Archive for

Organic Chemistry

Arkivoc 2019, part iv, 44-68

\title{
Progress toward the total synthesis of mirabalin isomers
}

Pierre-Georges Echeverria, ${ }^{a}$ Amandine Pons, ${ }^{a}$ Sébastien Prévost, ${ }^{a}$ Charlène Férard, ${ }^{a}$ Johan Cornil, ${ }^{b}$ Amandine Guérinot, ${ }^{b}$ Janine Cossy, ${ }^{* b}$ Phannarath Phansavath*a and Virginie Ratovelomanana-Vidal*a

a PSL University, Chimie ParisTech, CNRS, Institute of Chemistry for Life and Health Sciences (i-CLeHS) 75005 Paris, France

${ }^{b}$ Laboratoire de Chimie Organique, Institute of Chemistry, Biology and Innovation (CBI)-UMR 8231, ESPCI Paris/CNRS/PSL Research University, 10 rue Vauquelin 75231 Cedex 05 Paris, France

Email: janine.cossy@espci.fr, phannarath.phansavath@chimieparistech.psl.eu, virginie.vidal@chimieparistech.psl.eu

Dedicated to Prof. Stephen Hanessian for his outstanding contributions to the field of organic chemistry

Received 01-07-2019

Accepted 02-24-2019

Published on line 02-28-2019

\section{Abstract}

Key fragments of the cytotoxic marine macrolide mirabalin have been synthesized, by using a flexible strategy based on asymmetric reductions to control the hydroxy- and carbamate-bearing stereocenters. In particular, ruthenium or rhodium-mediated asymmetric hydrogenation and transfer hydrogenation were used in combination with a dynamic kinetic resolution to control two contiguous stereocenters in a single step.

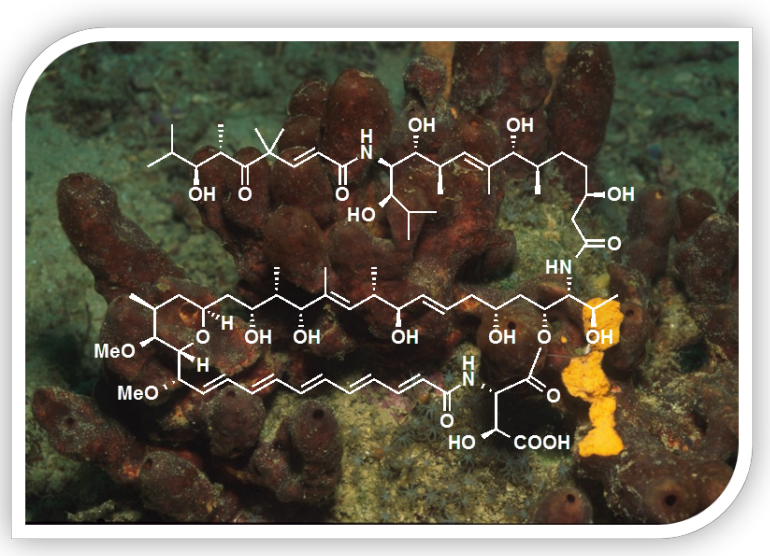

Keywords: Marine macrolides, asymmetric hydrogenation, asymmetric transfer hydrogenation, enantioselectivity, diastereoselectivity 


\section{Introduction}

Lithistid demosponges are an assemblage of sponges predominantly found in deeper waters. ${ }^{1,2}$ They are regarded as an excellent source of structurally diverse and bioactive secondary metabolites. ${ }^{3-5}$ In particular, numerous novel macrolides have been isolated from the Theonellidae family exhibiting excellent biological activities. ${ }^{6-7}$ Among them, mirabalin, a macrocycle, has been extracted from Siliquariaspongia mirabilis, a lithistid demosponge collected from the archipelago of Chuuk, Micronesia (Figure 1). ${ }^{8,9}$ This macrocycle exhibits potent cytotoxicity toward human colon tumor cell line HCT-116 with an IC 50 of $0.27 \mu \mathrm{M}$. Morever, it has been found that the macrocycle ring is crucial to mirabalin antitumor activity. Indeed, no inhibition of the tumor cells growth was observed when treated with the linear polyketide side-chain. From a structural point of view, mirabalin exhibits a 35-membered macrocyclic lactam-lactone possessing a fully conjugated pentaene system along with a tetrasubstituted tetrahydropyran ring. In addition, a linear polyketide side-chain is linked to the macrocyclic ring with an amide linkage. An investigation combining advanced NMR and MS techniques allowed the partial elucidation of the structure of mirabalin. Undeniably, because of the large number of stereocenters and the low amount of material available, the absolute configurations of 12 out of the 25 stereocenters could not be assigned. Nevertheless, the geometry for each of the double bonds was established unambiguously (Figure 1).
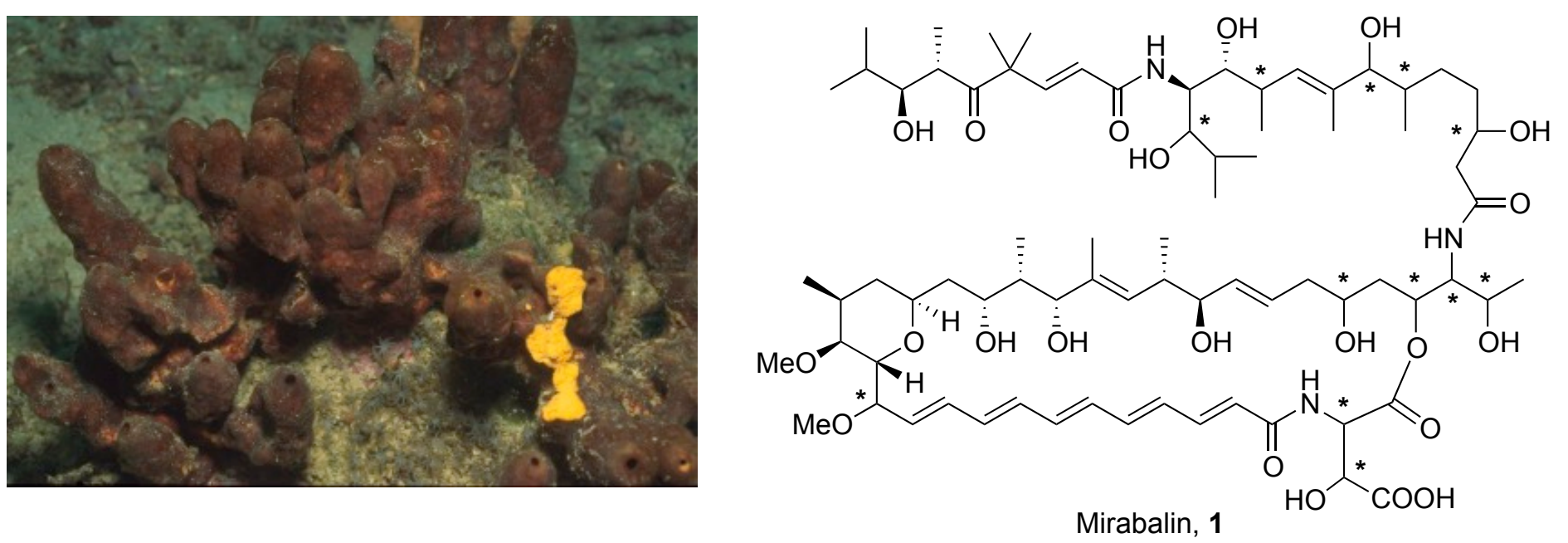

Figure 1. Picture of Siliquariaspongia mirabilis and the structure of mirabalin $(\mathbf{1})^{9}$.

As part of our ongoing studies on the total synthesis of natural products, ${ }^{10-17}$ and intrigued by the structural intricacy of this macrolide, we embarked on a study aiming at developing a flexible, convergent and modular route to one isomer of mirabalin where the configurations of the twelve unknown stereocenters were arbitrarily fixed (Scheme 1). Recently, we reported a synthetic strategy toward the C44-C65 polyketide side chain ${ }^{18}$ and toward the C14-C29 fragment of mirabalin, ${ }^{19}$ as well as a straightforward method to construct the pentaene moiety $\mathrm{C} 4-\mathrm{C} 14 .{ }^{20}$ Scheme 1 shows the mirabalin global retrosynthetic plan with late stage esterification, peptide coupling and $\mathrm{C}=\mathrm{C}$ bond formation to access the macrocycle by macrolactamization, suggesting $\mathbf{A}$ and $\mathbf{B}$ as suitable key intermediates for the sake of convergence. 


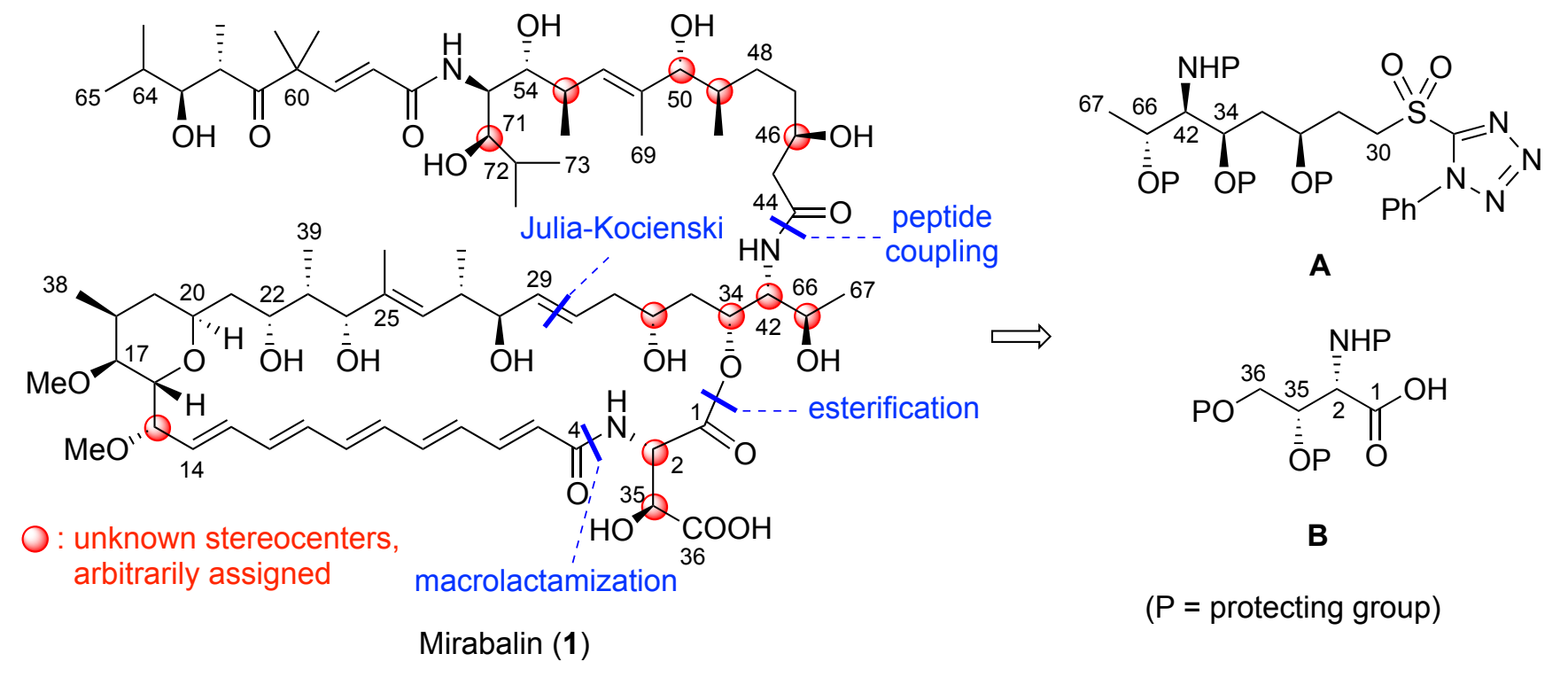

Scheme 1. Global retrosynthetic plan for mirabalin (1)

Herein, we report a flexible synthesis of fragments $\mathbf{A}$ and $\mathbf{B}$ but also details about the synthesis of isomers of the C44-C65 side chain of mirabalin.

\section{Results and Discussion}

\section{Synthesis of fragment $A$}

Our retrosynthetic analysis was based on known methods for the introduction of the phenyltetrazolyl sulfone moiety by a hydroboration/Mitsunobu/oxidation sequence applied to alkene $\mathbf{2}$. We therefore envisaged that the aldehyde $\mathbf{3}$ represented a useful intermediate in the synthesis of the unsaturated compound $\mathbf{2}$, and aldehyde 3 could be obtained from $\alpha$-amino $\beta$-ketoester 4 after asymmetric hydrogenation ${ }^{21-23}$ via a dynamic kinetic resolution, ${ }^{24-26}$ Claisen condensation and asymmetric hydrogenation (Scheme 3). ${ }^{27-29}$

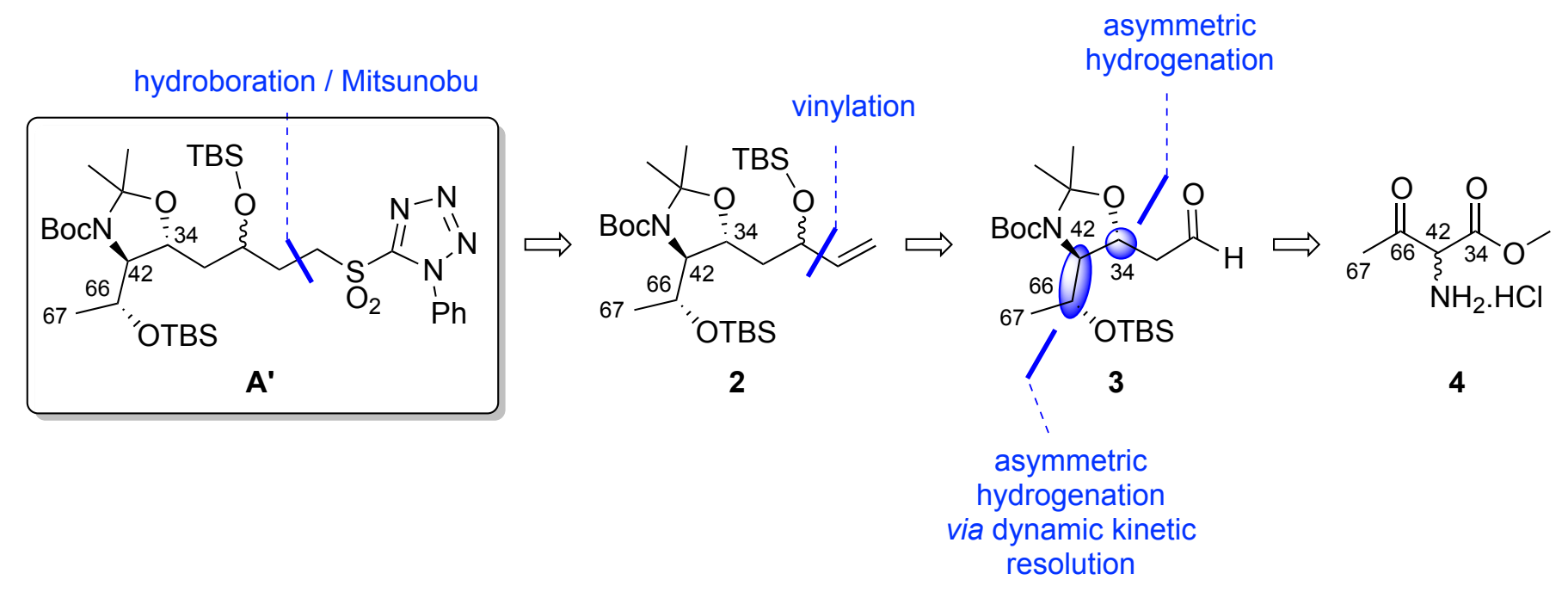

Scheme 2. Retrosynthetic analysis of fragment $\mathbf{A}$ (compound $\mathbf{A}^{\prime}$ ). 
Our synthesis began with the preparation of the hydrochloride ammonium salt $\mathbf{4}$ in two steps from methyl acetoacetate 5 after nitrosation followed by hydrogenolysis of the oxime 6 under acidic conditions. To control the anti configuration at $\mathrm{C} 66$ and $\mathrm{C} 42$, a ruthenium-catalyzed asymmetric hydrogenation through dynamic kinetic resolution of the racemic $\alpha$-amino $\beta$-keto ester hydrochloride 4 was envisaged. Using this method, the corresponding anti-aminoalcohol was successfully prepared with $90 \%$ diastereomeric excess and $91 \%$ enantiomeric excess. The reaction was performed in $\mathrm{CH}_{2} \mathrm{Cl}_{2} / \mathrm{MeOH}(10: 1)$ at $50{ }^{\circ} \mathrm{C}$ under 13 bar of $\mathrm{H}_{2}$ using 2 mol\% of the in situ prepared [Ru((R)-Synphos)] $\left.\mathrm{Br}_{2}\right]$ catalyst ${ }^{30}$ (Scheme 3 ). Protection of the amino group as a carbamate and conversion of the hydroxy function into a TBS ether delivered compound 8 . The ensuing fully protected amino hydroxy ester $\mathbf{8}$ was then submitted to a Claisen condensation with tert-butyl acetate under basic conditions to deliver $\beta$-keto ester 9 in $87 \%$ yield (Scheme 3 ).
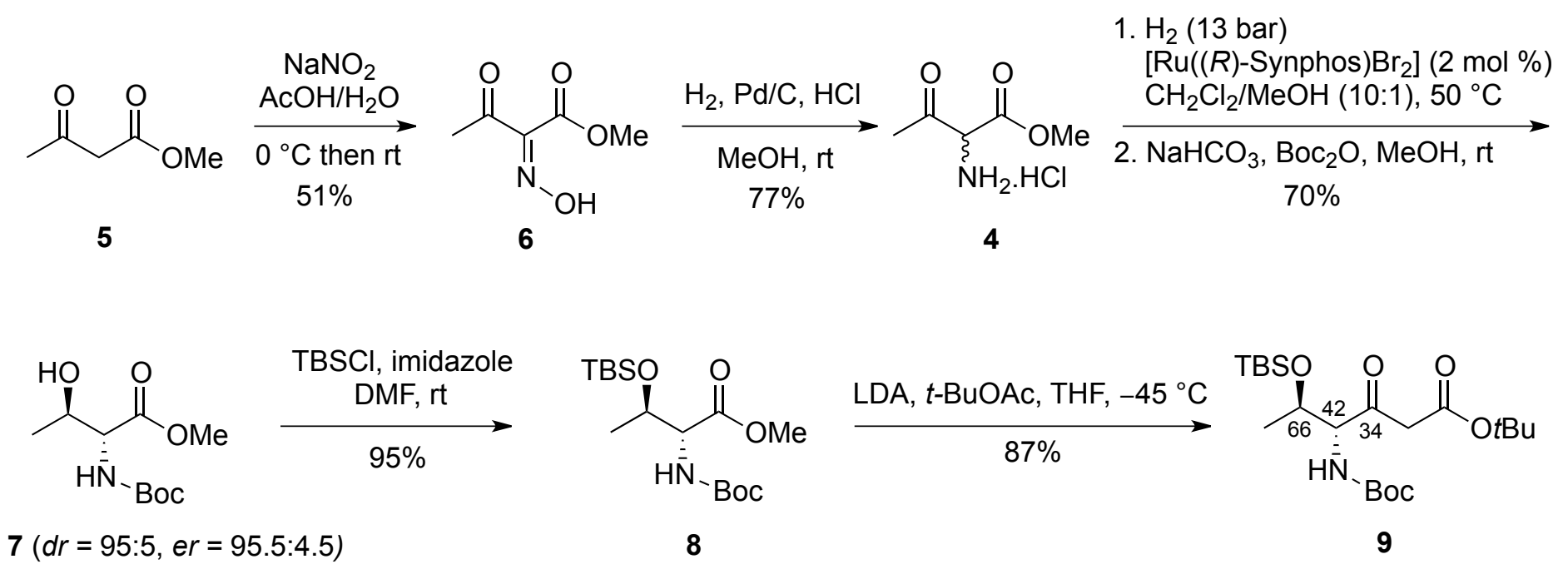

Scheme 3. Preparation of $\beta$-keto ester 9.

We then turned our attention to the asymmetric hydrogenation of ketone 9 (Scheme 4). Different conditions varying catalyst loading and hydrogen pressure were screened using the binuclear complex $\left[\{\mathrm{RuCl}((S)-S y n p h o s)\}_{2}(\mu-\mathrm{Cl})_{3}\right]\left[\mathrm{Me}_{2} \mathrm{NH}_{2}\right]^{30}$ in $\mathrm{EtOH}$ at $50^{\circ} \mathrm{C}$. No influence of the catalyst loading was observed on the stereochemical outcome of the reaction. However, changing the hydrogen pressure from 70 to 100 bar afforded a better result in terms of diastereoselectivity ( $d r$ switching from 74:26 to $>99: 1$ ). With these optimized conditions in hand, the synthesis was pursued with the protection of aminoalcohol 10 to the oxazolidine 11. It was found that the starting material $\mathbf{1 0}$ was not fully converted during the reaction and the acidic conditions led to the cleavage of the TBS group on both compounds $\mathbf{1 0}$ and $\mathbf{1 1}$. The yield was modest and variable despite several experimentations. Nevertheless, compound $\mathbf{1 1}$ was converted to aldehyde $\mathbf{3}$ with Dibal-H in $77 \%$ yield. Addition of vinylmagnesium bromide to aldehyde 3 at low temperature provided the desired allylic alcohol 12 in 77\% yield with a diastereoselectivity of 62:38. The two stereoisomers were separated by flash column chromatography and the synthesis of compound $\mathbf{A}^{\prime}$ was carried out with the major diastereomer, the absolute configuration of which was determined by Mosher analysis (Scheme 4). ${ }^{31}$ 


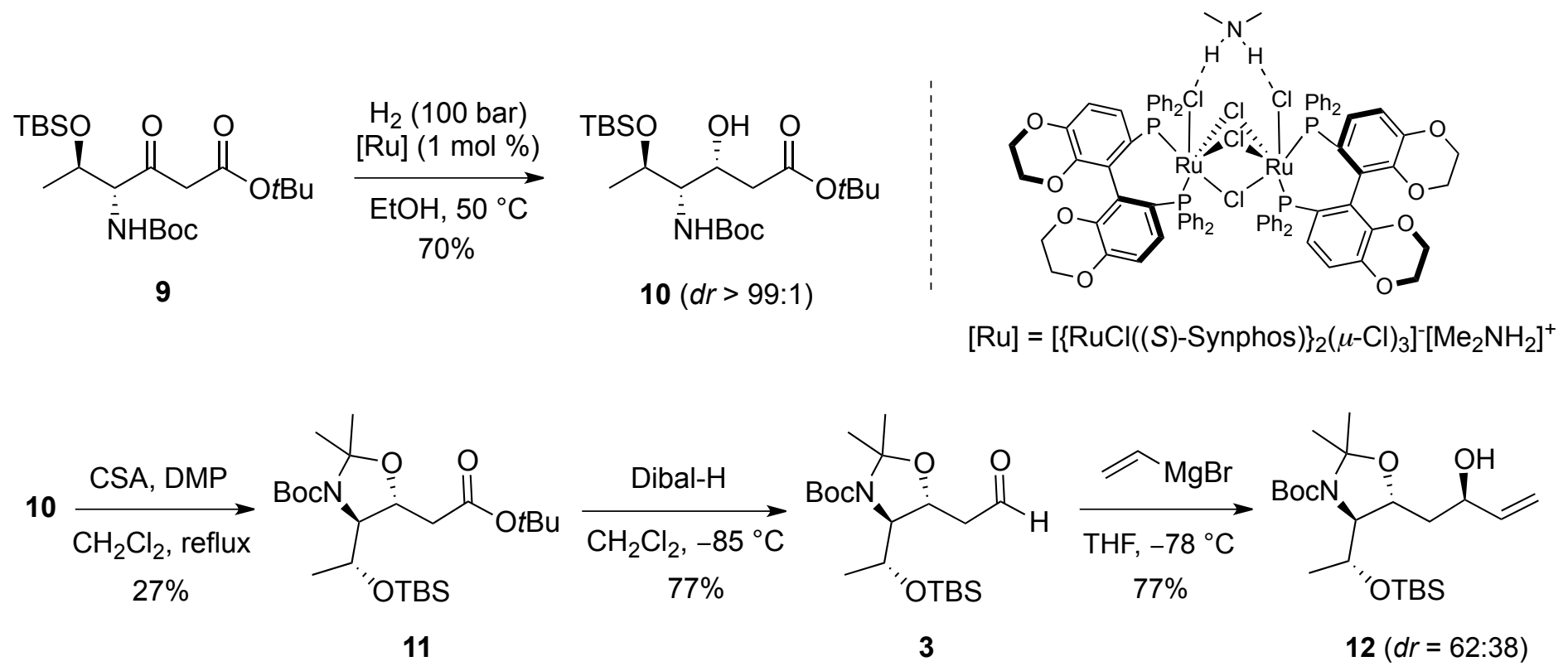

Scheme 4. Preparation of alcohol 12.

After protection of alcohol 12 (TBSCl, imid, DMF, rt), hydroboration/oxidation of the obtained compound 2 furnished the desired product 13 in 75\% yield. Formation of thioether 14 was accomplished using a Mitsunobu reaction $^{32,33}$ applied to alcohol 13 (1-phenyl-1H-tetrazole-5-thiol, DIAD, PPh, THF, rt) and the thioether 14 was converted to the corresponding sulfone in $68 \%$ yield by oxidation with $m$-CPBA (Scheme 5 ). Thus, compound $\mathbf{A}^{\prime}$ was obtained in 14 steps in 1.1\% overall yield from methyl acetoacetate 5 and three contiguous stereocenters were controlled using asymmetric hydrogenation. To appreciate the feasibility of our envisaged retrosynthetic plan, we performed a Julia-Kocienski olefination ${ }^{34}$ between compound $\mathbf{A}^{\prime}$ and the aldehyde generated from alcohol $15^{35}$ (corresponding to the C25-C28 fragment of the macrocycle, Scheme 1). Pleasingly, the desired $E$-alkene was obtained in $76 \%$ yield with tight control of the double bond geometry $(E / Z>95: 5)$ (Scheme 5). 


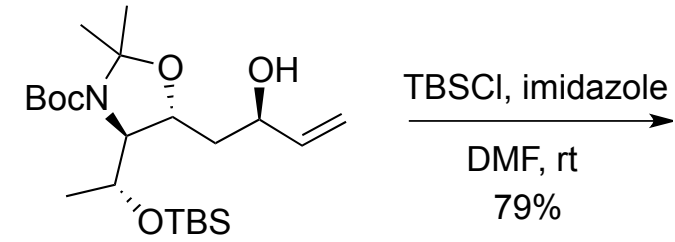

12

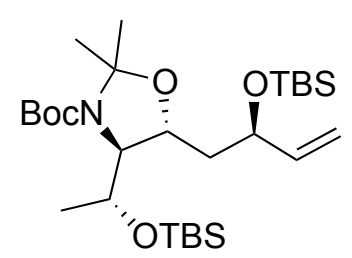

2
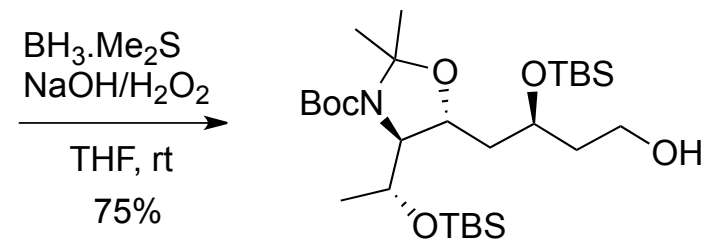

13

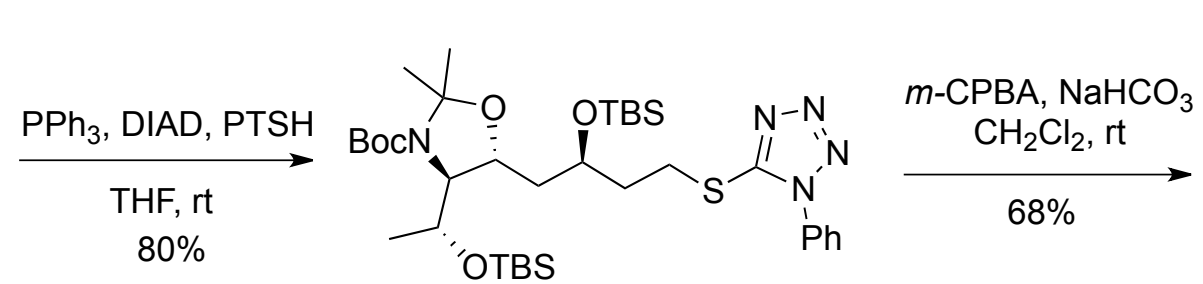

14

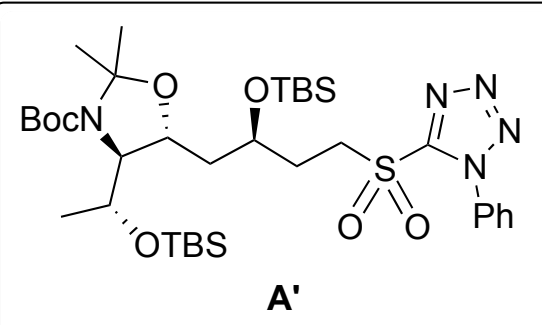

$A^{\prime}$
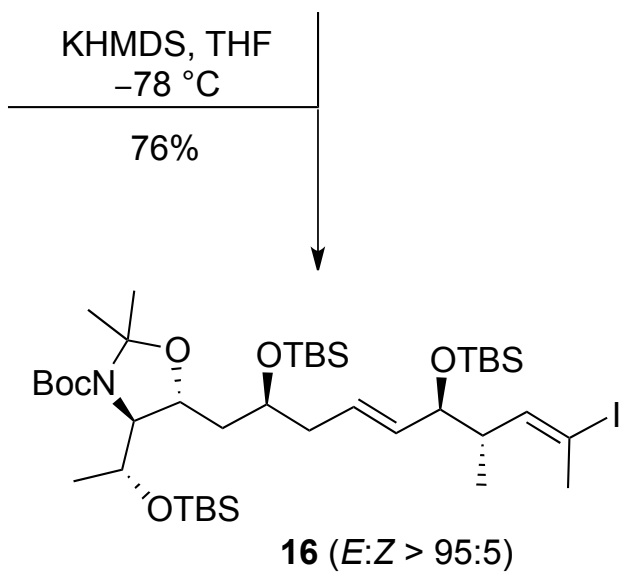

Scheme 5. Synthesis of compound $\mathbf{A}^{\prime}$ and subsequent Julia-Kocienski olefination.

\section{Synthesis of fragment B}

The retrosynthetic analysis for the construction of the second major building block B is summarized in Scheme 6 for compound B'. Our strategy focused on the flexibility in term of stereochemistry. For this purpose, asymmetric hydrogenation and transfer hydrogenation ${ }^{36-40}$ were chosen as complementary methods to access all four stereoisomers of compound B'.

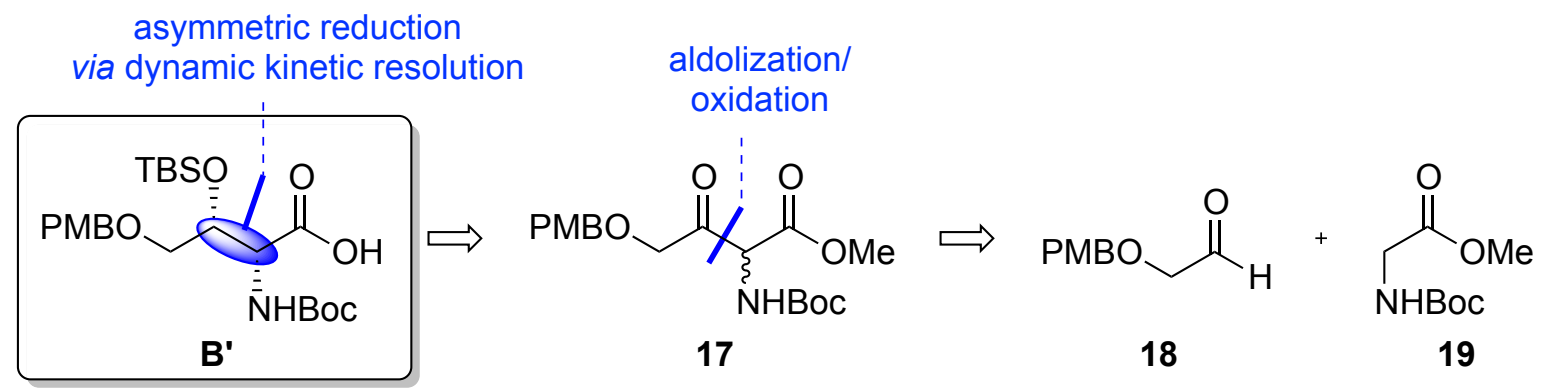

Scheme 6. Retrosynthetic analysis of fragment B (compound B'). 
The synthesis of the $\alpha$-carbamate $\beta$-ketoester $\mathbf{1 7}$ proceeded as shown in Scheme 7. After PMBmonoprotection of ethane-1,2-diol 20 and Dess-Martin oxidation of the obtained 21, aldehyde $\mathbf{1 8}$ was isolated in $61 \%$ yield. The other partner 19 was prepared by simple $N$-Boc protection of glycine methyl ester hydrochloride $\mathbf{2 2}$ in $\mathbf{9 7 \%}$ yield. Thereafter, reaction of the zinc derivative of $\mathbf{1 9}$ with aldehyde $\mathbf{1 8}$ furnished the corresponding $\alpha$-carbamate $\beta$-hydroxy ester whose oxidation afforded the desired $\beta$-keto ester $\mathbf{1 7}$. It is noteworthy that, after thorough investigation, only the use of Dess-Martin periodinane in the absence of $\mathrm{NaHCO}_{3}$ allowed the oxidation, albeit in low yield; other attempts using IBX, Parikh-Doering, Ley-Griffith, Swern, Fétizon or pyridinium dichromate reagents led only to degradation products. With the desired keto ester $\mathbf{1 7}$ in hand, we focused on the asymmetric reduction of $\mathbf{1 7}$ via dynamic kinetic resolution.

After careful optimization of the reaction parameters, compound syn-23 was obtained in $\mathbf{7 3 \%}$ yield with good diastereomeric ratio ( $d r=86: 14)$ and enantioselectivity ( $e r=90: 10)$ by using $0.5 \mathrm{~mol} \%$ of the complex $\left[\operatorname{RuBr}_{2}\left((S)\right.\right.$-Synphos)] prepared in situ from $\left[\operatorname{Ru}(C O D)(2-\text { methylallyl })_{2}\right],{ }^{30}$ under 50 bar of hydrogen pressure at $50{ }^{\circ} \mathrm{C}$ in dichloromethane. Subsequent TBS protection of the hydroxyl gave syn-24 and the desired compound syn-B' was finally obtained by hydrolysis of the methyl ester to the corresponding carboxylic acid (Scheme 7).

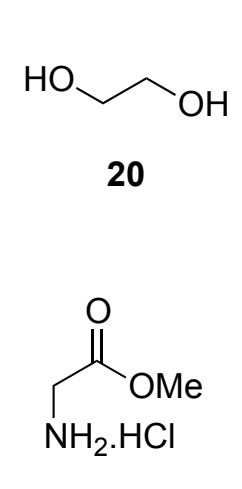

22

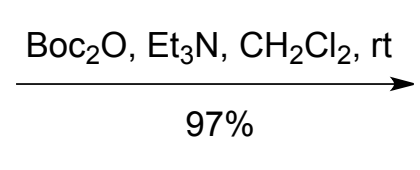

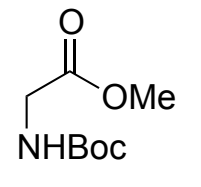

19
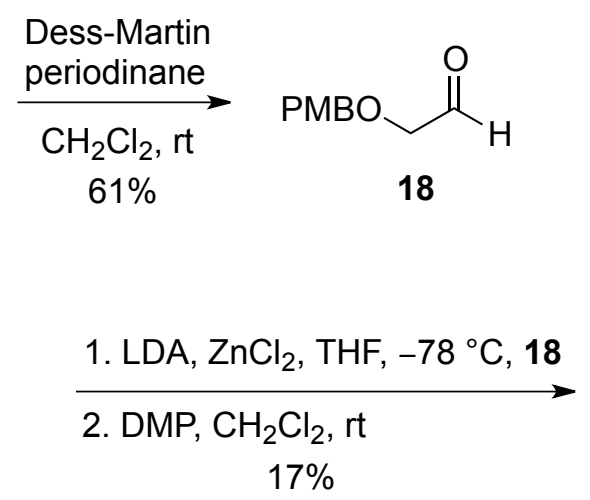

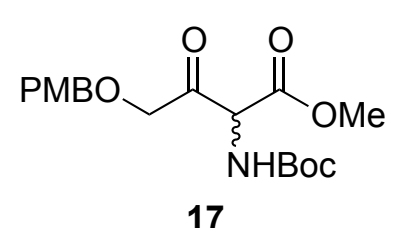

17

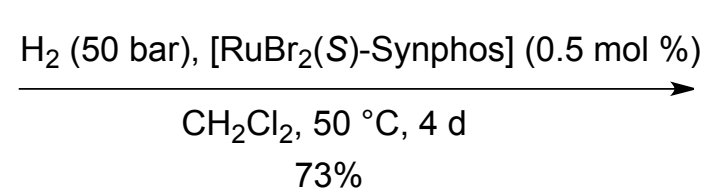

$73 \%$

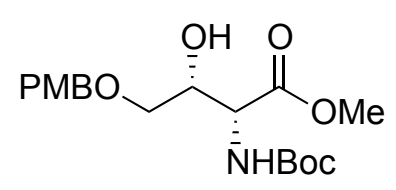

syn-23 $(d r=86: 14$, er $=90: 10)$

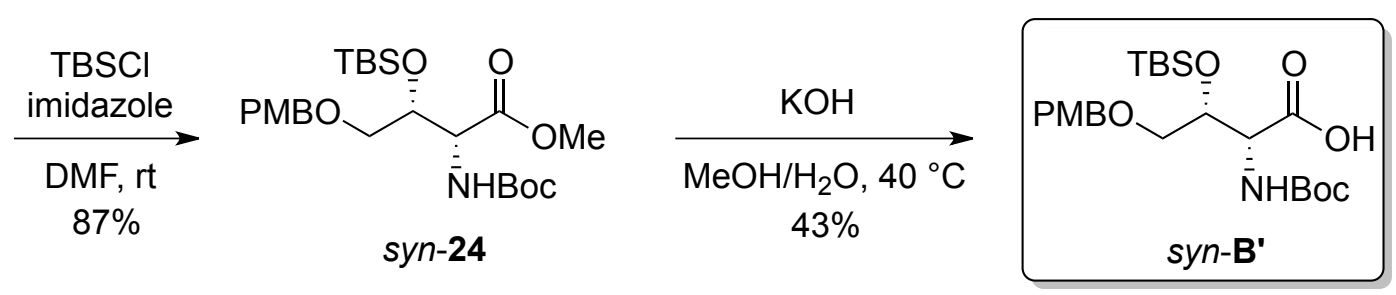

Scheme 7. Synthesis of compound syn-B' through asymmetric hydrogenation.

To obtain the anti-isomer of fragment B, we turned our attention to the use of asymmetric transfer hydrogenation of $\mathbf{1 7}$ in combination with dynamic kinetic resolution. Preliminary screening employing different catalysts Cat I, ${ }^{41}$ Cat II, ${ }^{42}$ and Cat III ${ }^{43,44}$ with $\mathrm{HCOOH} / \mathrm{Et}_{3} \mathrm{~N}(5: 2)$ as the hydrogen donor demonstrated that faster reaction times as well as better diastereoselectivities in favor of compound anti-23 $(d r=78: 22)$ were obtained with catalyst Cat III. Moreover, the catalytic charge could be decreased from 2 mol\% to 0.1 
mol\% without any loss of stereoselectivity and compound $\mathbf{2 3}$ was isolated with a comparable $\mathbf{7 0 \%}$ yield. It is noteworthy that at this point of the synthesis inseparable mixtures of syn- and anti-isomers were obtained. To complete the synthesis of fragment $\mathbf{B}$, the newly formed hydroxy-ester $\mathbf{2 3}$ was protected by using TBSCl and imidazole in $51 \%$ yield before hydrolysis of the ester with TMSOK yielded the fully protected compound antiB'. Gratifyingly, flash chromatography allowed separation of the syn- and anti-isomers (Scheme 8).
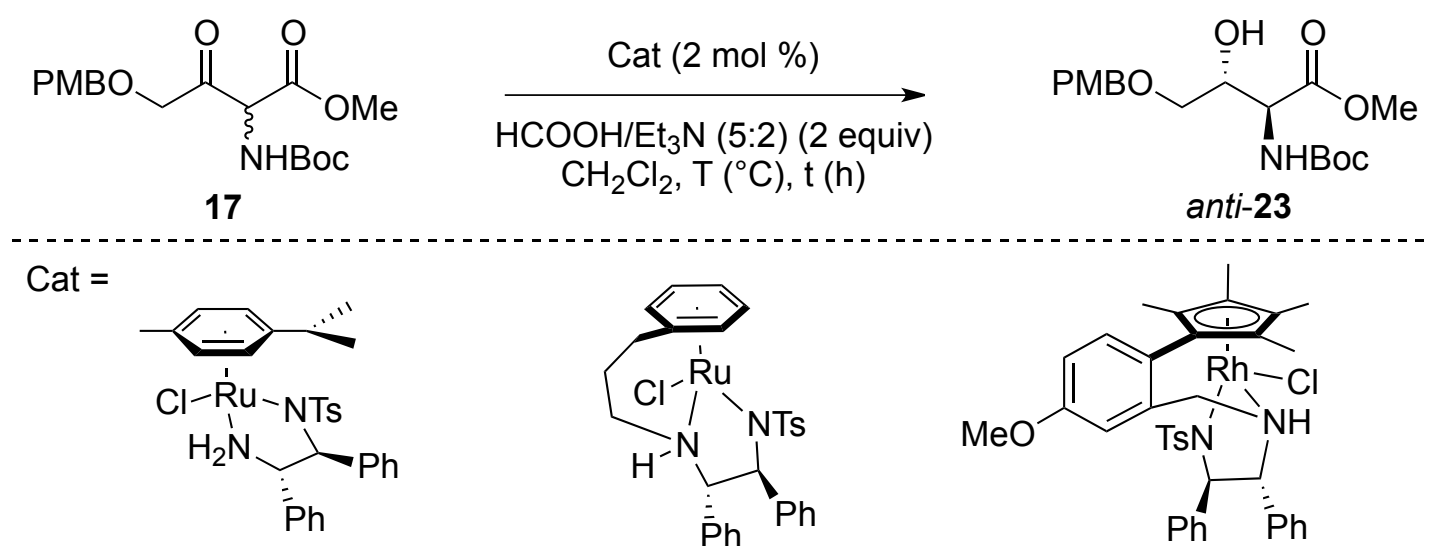

Cat I

$\mathrm{rt}, 25 \mathrm{~h}$ then $40{ }^{\circ} \mathrm{C}, 24 \mathrm{~h}$ $71 \%(d r=62: 38$, er $=96: 4)$

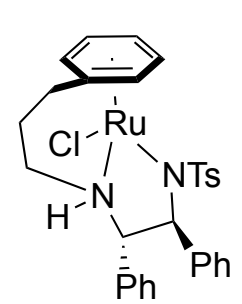

Cat II

$$
\begin{array}{cc}
\text { rt, } 1 \mathrm{~h} & \mathrm{rt}, 45 \mathrm{~min} \\
69 \%(d r=58: 42, \text { er }=88: 12) \quad 74 \%(d r=78: 22, \text { er }=97: 3)
\end{array}
$$

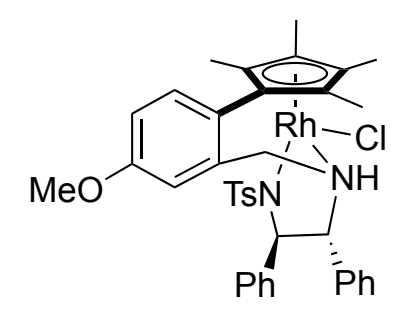

Cat III

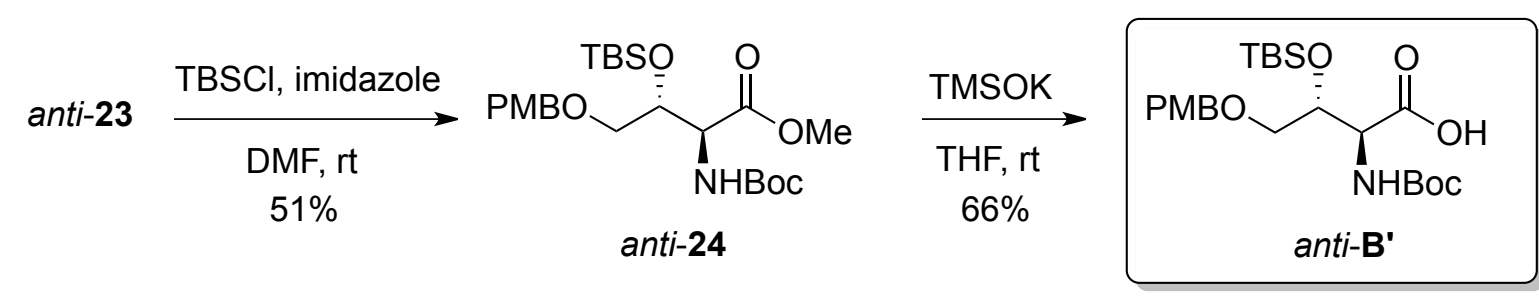

Scheme 8. Synthesis of compound anti-B' through asymmetric transfer hydrogenation.

Thus, compound anti-B' was synthesized in 7 steps starting from ethylene glycol 20 in $2.2 \%$ overall yield. This strategy inherently makes the synthesis flexible because all the four stereoisomers of fragment $\mathbf{B}$ can be synthesized by simply changing the configuration of the chiral ligand and by moving from asymmetric hydrogenation to asymmetric transfer hydrogenation.

\section{Synthesis of stereoisomers of the C44-C65 side chain}

As outlined above, flexibility of our synthetic plan was essential as the configurations of several stereogenic centers remain unknown. We decided to prepare other isomers of the north fragment of the targeted molecule (C44-C65 fragment, the two relevant stereocenters are highlighted with a star on Scheme 9). As such, it was shown previously that the C44-C65 part of the molecule could easily be synthesized after assembly of fragments C1, C2 and C3 (Scheme 9). ${ }^{18}$ Herein, we focused our attention to the modifications of the two C46 and C49 stereocenters of fragment C3. The stereogenic center at C46 would be easily modulated by simply changing the configuration of the upstream Roche ester, while modification of the configuration at the C49 stereocenter would be achieved by switching the ligand configuration during the asymmetric hydrogenation, thus allowing access to new isomers of the side chain. 


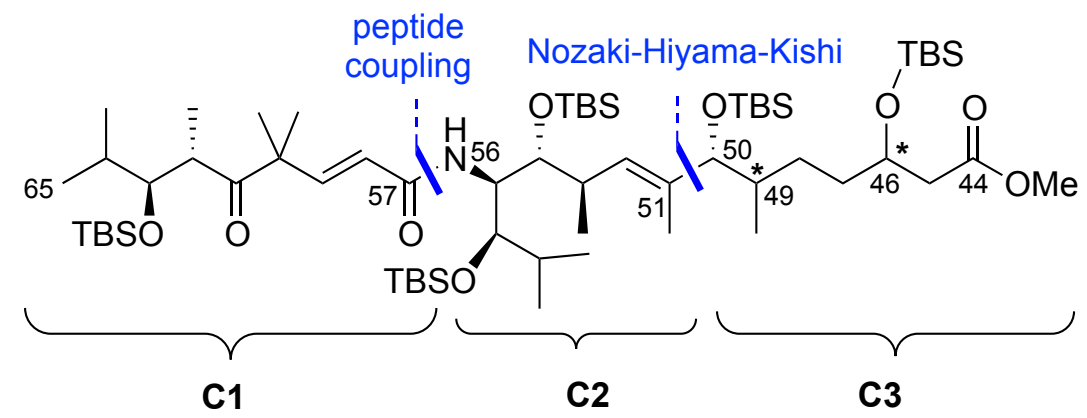

Scheme 9. Retrosynthetic analysis of mirabalin side chain C44-C65.

The synthesis began with the benzyl protection of $(R)$-Roche ester $\mathbf{2 5}$ followed by reduction of the resulting ester 26 to 27 using $\mathrm{LiAlH}_{4}$. Garegg iodination ${ }^{45}\left(\mathrm{I}_{2}, \mathrm{PPh}_{3}\right.$, imid, $\left.\mathrm{CH}_{2} \mathrm{Cl}_{2}, \mathrm{rt}\right)$ of the latter afforded iodide 28, which was used for the alkylation of methyl acetoacetate ${ }^{46}$ to provide the key $\beta$-keto ester 29 (Scheme 10). With convenient amounts of compound $\mathbf{2 9}$ in hand, ruthenium-catalyzed asymmetric hydrogenation of the ketone was tackled. This transformation was accomplished using the in situ generated chiral complexes either $\left[\operatorname{RuBr}_{2}\left((S)\right.\right.$-Synphos)] or $\left[\operatorname{RuBr}_{2}((R)\right.$-Synphos)]. The stereochemical outcome of the reaction was controlled by choosing the appropriate configuration of the Synphos ligand and the reaction afforded $(S, S)$-30 and $(R, S)$-30 in $87 \%$ and $93 \%$ yields, respectively, as single diastereomers. The protection of these alcohols (TBSOTf, 2,6lutidine, $\left.\mathrm{CH}_{2} \mathrm{Cl}_{2}, 0^{\circ} \mathrm{C}\right)$ yielded $(S, S)$-31 and $(R, S)$-31 which were subjected to debenzylation conditions $\left(\mathrm{H}_{2}\right.$, $\mathrm{Pd} / \mathrm{C})$ to give $(S, S)$-32 and $(R, S)$-32, and subsequent Dess-Martin oxidation afforded aldehydes $(S, S)$-33 and $(R, S)$-33 required for the Nozaki-Hiyama-Kishi ${ }^{47,48}$ coupling with $(E)$-iodoalkene $34 .^{18}$ This reaction was performed in DMSO using $\mathrm{CrCl}_{2}$ (14 equiv) in the presence of a catalytic amount of $\mathrm{NiCl}_{2}(\mathrm{dppe})$ (6 mol\%). Under these conditions, alcohols $\mathbf{3 5}$ and $\mathbf{3 6}$ were obtained in $25 \%$ and $34 \%$ yields, respectively (quantitative yields based on the recovered starting material) as a mixture of diastereomers ( $d r=75: 25$ for $\mathbf{3 5}, d r=71: 29$ for 36) which were separated by flash chromatography on silica gel. 


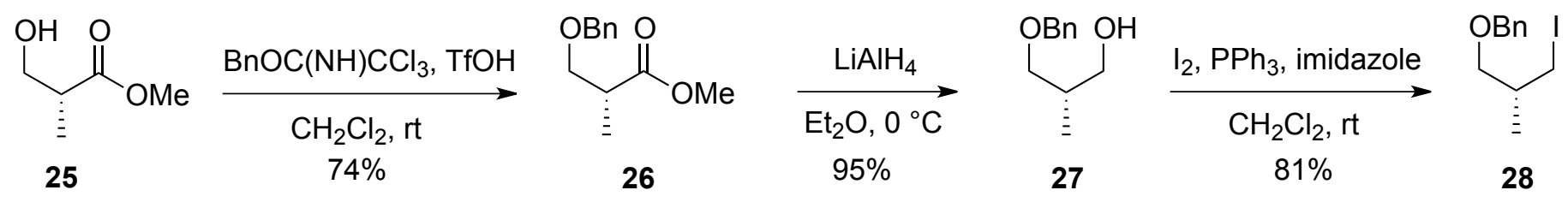

$\mathrm{H}_{2}$ (11 bar) [RuBr $2((S)-S y n p h o s)]$

or $\left[\operatorname{RuBr}_{2}((R)-S y n p h o s)\right]$

$(1 \mathrm{~mol} \%)$

$\mathrm{MeOH}, 50^{\circ} \mathrm{C}$<smiles>[R]C([R])(CC[C@@H](C)COc1ccccc1)CC(=O)OC</smiles>

$(S, S)-\mathbf{3 0}, \mathrm{R}^{1}=\mathrm{H}, \mathrm{R}^{2}=\mathrm{OH}(87 \%)$ or $(R, S)-30, \mathrm{R}^{1}=\mathrm{OH}, \mathrm{R}^{2}=\mathrm{H}(93 \%)$

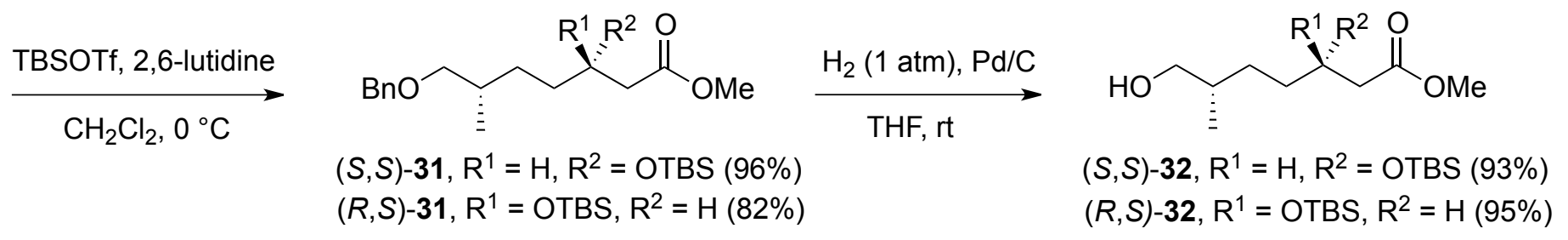

$\underset{\mathrm{CH}_{2} \mathrm{Cl}_{2}, \mathrm{rt}}{\stackrel{\text { Dess-Martin periodinane }}{\longrightarrow}}$

$(S, S)-33, \mathrm{R}^{1}=\mathrm{H}, \mathrm{R}^{2}=$ OTBS $(67 \%)$

$(R, S)-33, \mathrm{R}^{1}=$ OTBS, $\mathrm{R}^{2}=\mathrm{H}(64 \%)$

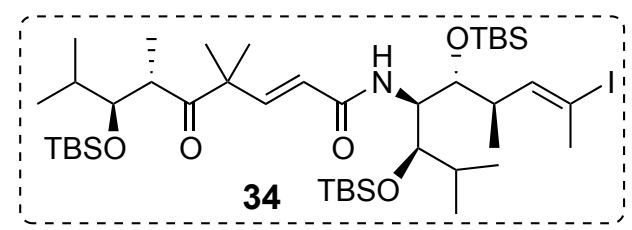

34, $\mathrm{CrCl}_{2}, \mathrm{NiCl}_{2}(\mathrm{dppe})$
DMSO, $\mathrm{rt}$

$34 \%$

34, $\mathrm{CrCl}_{2}, \mathrm{NiCl}_{2}$ (dppe) DMSO, rt
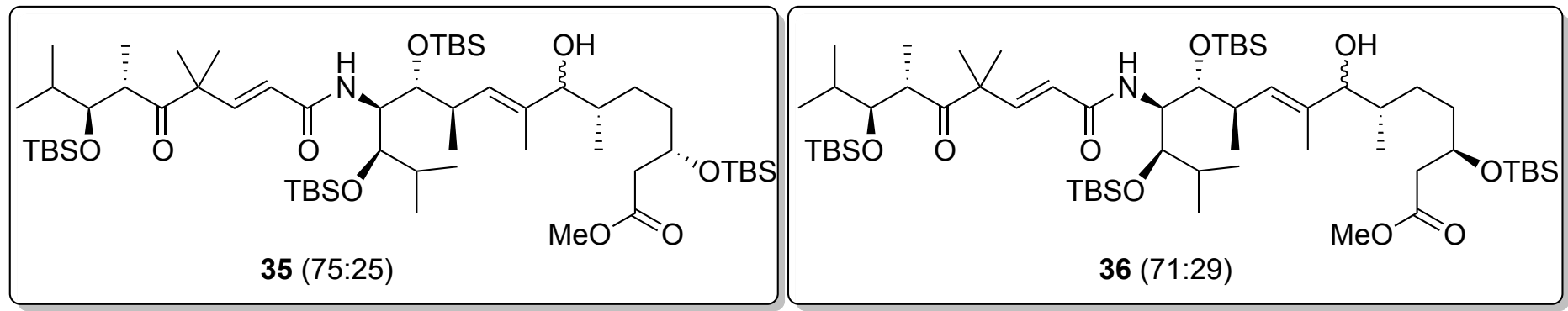

Scheme 10. Synthesis of stereoisomers $\mathbf{3 5}$ and $\mathbf{3 6}$ of the C44-C65 side chain of mirabalin.

In summary, the facile synthesis of two new isomers 35 and 36 of the C44-C65 part of mirabalin demonstrated the high flexibility of the synthetic plan offered by using asymmetric reduction and one can envisage to access other stereoisomers using this versatile reduction. 


\section{Conclusions}

In conclusion, the synthesis of several key fragments of mirabalin 1 was achieved using flexible and robust methods based on asymmetric hydrogenation and transfer hydrogenation. The C30-C67 fragment (compound $\mathbf{A}^{\prime}$ ) as well as two isomers of the C1-C36 subunits (compounds syn-B' and anti-B') have been prepared. Moreover the synthesis of two isomers of the C44-C65 part of mirabalin has been achieved. Most importantly, the flexibility of this approach provides a platform for modification of several unknown stereocenters. The assembly of the different fragments is currently being pursued in our laboratory.

\section{Experimental Section}

General. All air and/or water sensitive reactions were carried out under an argon atmosphere. Tetrahydrofuran and diethyl ether were distilled from sodium-benzophenone. Dichloromethane was distilled from calcium hydride. Reactions were monitored by thin layer chromatography carried out on precoated silica gel plates (E. Merck ref. $555460 \mathrm{~F} 254$ ) and revealed with either an ultra-violet lamp ( $\lambda=254 \mathrm{~nm}$ ), a potassium permanganate solution ( $3 \mathrm{~g} \mathrm{KMnO}_{4}, 20 \mathrm{~g} \mathrm{~K}_{2} \mathrm{CO}_{3}, 0.25 \mathrm{~mL} \mathrm{AcOH}, 300 \mathrm{~mL} \mathrm{H} \mathrm{O}$ ), a ninhydrin solution (1 g ninhydrin, $100 \mathrm{~mL} \mathrm{EtOH} / \mathrm{H}_{2} \mathrm{SO}_{4}: 95 / 5$ ) or a Kagi-Mosher solution ( $8 \mathrm{~mL} p$-anisaldehyde, $16 \mathrm{~mL} \mathrm{H} \mathrm{SO}_{4}, 800 \mathrm{~mL}$ $\mathrm{AcOH})$. The nuclear magnetic resonance spectra were recorded on a Bruker $\mathrm{AC} 300\left({ }^{1} \mathrm{H}: 300 \mathrm{MHz},{ }^{13} \mathrm{C}: 75 \mathrm{MHz}\right)$ or an Avance $400\left({ }^{1} \mathrm{H}: 400 \mathrm{MHz},{ }^{13} \mathrm{C}: 100 \mathrm{MHz}\right)$ instrument. Data are reported as follows: chemical shifts $(\delta)$, multiplicity (recorded as s singlet; $d$, doublet; t, triplet; q, quartet; quint, quintet; sxt, sextet; sep, septet; $m$, multiplet; dd, doublet of doublets; dt, doublet of triplets; td, triplet of doublets; br, broad), integration and coupling constants. Melting points $(\mathrm{mp})$ were determined on a Büchi apparatus and are uncorrected. Optical rotations were measured on a Perkin-Elmer 241 polarimeter. High resolution mass spectrometric (HRMS) analyses were measured on LTQ-Orbitrap (Thermo Fisher Scientific) at Pierre et Marie Curie University. Enantiomeric excesses were determined by HPLC using a chiral stationary phase column (Chiralpak IA) and eluting with a hexane/isopropanol mixture as indicated.

Methyl (Z)-2-(hydroxyimino)-3-oxobutanoate (6). A solution of methyl acetoacetate 5 (15 g, $129 \mathrm{mmol}, 1$ equiv) in acetic acid $\left(100 \mathrm{~mL}\right.$ ) was cooled to $0{ }^{\circ} \mathrm{C}$ and a suspension of $\mathrm{NaNO}_{2}(22.3 \mathrm{~g}, 323 \mathrm{mmol}, 2.5$ equiv) in water $\left(75 \mathrm{~mL}\right.$ ) was added dropwise, the temperature of the reaction mixture being kept below $5{ }^{\circ} \mathrm{C}$. After evolution of the brown fumes, the stirring was maintained $1.5 \mathrm{~h}$ at $0{ }^{\circ} \mathrm{C}$ and then at $\mathrm{rt}$ until completion of the reaction, monitored by TLC $(1 \mathrm{~h})$. The reaction mixture was then diluted and extracted with $\mathrm{Et}_{2} \mathrm{O}$. The combined organic layers were washed with saturated aqueous $\mathrm{NaHCO}_{3}$, dried over $\mathrm{MgSO}_{4}$, concentrated under reduced pressure and the crude mixture was purified by silica gel column chromatography (cyclohexane/EtOAc: $6 / 4)$ to give the desired product $6(9.54 \mathrm{~g}, 51 \%)$ as a white solid. $\mathrm{mp} 39{ }^{\circ} \mathrm{C} ; \mathrm{R}_{f}$ (cyclohexane/EtOAc: 8/2) 0.17; IR (film): 3353, 1742, 1681, 1415, 1317, 1244, 1070, $1000 \mathrm{~cm}^{-1}$; ${ }^{1} \mathrm{H}$ NMR (300 $\left.\mathrm{MHz}, \mathrm{CDCl}_{3}\right) \delta 9.33(\mathrm{~s}, 1 \mathrm{H}), 3.91(\mathrm{~s}, 3 \mathrm{H}), 2.41(\mathrm{~s}, 3 \mathrm{H}) .{ }^{13} \mathrm{C} \mathrm{NMR}\left(75 \mathrm{MHz}, \mathrm{CDCl}_{3}\right) \delta 193.9,162.1,151.0,52.8,25.3$.

Methyl 2-amino-3-oxobutanoate hydrochloride (4). To a solution of oxime 6 (7.34 g, $51 \mathrm{mmol}, 1$ equiv) in $\mathrm{MeOH}(126 \mathrm{~mL}$ ) was added Pd/C 10\% (1.79 g, $1.68 \mathrm{mmol}, 0.033$ equiv). $2 \mathrm{~N} \mathrm{HCl}$ in Et $2 \mathrm{O}(84.2 \mathrm{~mL}, 168.3 \mathrm{mmol}$, 3.3 equiv) was added dropwise to the resulting mixture and the argon atmosphere was replaced with hydrogen (balloon). The reaction mixture was stirred at $\mathrm{rt}$ under atmospheric pressure of hydrogen for $24 \mathrm{~h}$ (reaction monitored by TLC). The suspension was then filtered on a celite pad and washed with $\mathrm{MeOH}$. The filtrate was concentrated under reduced pressure and the yellow solid was washed with EtOAc to give the title 
compound 4 as a white solid (6.57 g, 77\%). mp $148{ }^{\circ} \mathrm{C}$; IR (film): 3419, 2986, 1745, 1727, 1469, 1279, 1241, $1164,1131,1089,878 \mathrm{~cm}^{-1} ;{ }^{1} \mathrm{H}$ NMR $(300 \mathrm{MHz}$, DMSO-d $) \delta 9.08(\mathrm{~s}, 3 \mathrm{H}), 5.26(\mathrm{~s}, 1 \mathrm{H}), 3.79(\mathrm{~s}, 3 \mathrm{H}), 2.38(\mathrm{~s}, 3 \mathrm{H})$; ${ }^{13} \mathrm{C}$ NMR $\left(75 \mathrm{MHz}\right.$, DMSO- $\left.d_{6}\right) \delta 196.7,164.2,61.2,53.6,28.2$.

Methyl (2R,3R)-2-[(tert-butoxycarbonyl)amino]-3-hydroxybutanoate (7). (R)-Synphos (377 mg, $0.59 \mathrm{mmol}$, 0.022 equiv) and $\left[\mathrm{Ru}(\mathrm{COD})\left(\eta^{3} \text {-2-methylallyl }\right)_{2}\right](171 \mathrm{mg}, 0.54 \mathrm{mmol}, 0.02$ equiv) were placed in a reaction tube and the vessel was purged with argon. Anhydrous acetone $(20 \mathrm{~mL})$ previously degassed by three vacuumargon cycles was added at rt. To this suspension was added dropwise methanolic $\mathrm{HBr}(6.6 \mathrm{~mL}, 0.044$ equiv of a $0.18 \mathrm{~N}$ solution prepared by adding $48 \%$ aqueous $\mathrm{HBr}$ in degassed methanol) and the suspension was stirred at room temperature for $30 \mathrm{~min}$. The suspension immediately turned yellow, then an orange precipitate appeared and the solvent was thoroughly evaporated under vacuum to give the catalyst as an orange-brown solid $\left[\operatorname{RuBr}_{2}((R)\right.$-Synphos)], which was used directly. The $\beta$-keto ester hydrochloride 4 (4.5 g, $26.9 \mathrm{mmol}, 1$ equiv) was then added followed by previously degassed anhydrous $\mathrm{CH}_{2} \mathrm{Cl}_{2}(20 \mathrm{~mL})$ and degassed alcoholic solvent $\mathrm{MeOH}(2 \mathrm{~mL})$. The reaction vessel was degassed by three vacuum-argon cycles and then placed under argon in a $250 \mathrm{~mL}$ stainless steel autoclave. The argon atmosphere was replaced with hydrogen by three cycles of pressurizing and the pressure adjusted to 13 bar. The autoclave was heated at $50{ }^{\circ} \mathrm{C}$ and stirring was maintained for $23 \mathrm{~h}$. After cooling, the reaction mixture was concentrated under reduced pressure to afford the crude $\beta$-hydroxy ester. It was then dissolved in degassed $\mathrm{MeOH}(50 \mathrm{~mL})$ and $\mathrm{NaHCO}_{3}(9.02 \mathrm{~g}, 107.4 \mathrm{mmol}$, 4 equiv) and di-tert-butyl dicarbonate $(5.86 \mathrm{~g}, 26.9 \mathrm{mmol}, 1$ equiv) was added. The mixture was stirred under ultrasonic irradiation during $4 \mathrm{~h}$. The solvent was removed under reduced pressure and the crude mixture was purified by silica gel column chromatography (petroleum ether/EtOAc: 6/4) to give the desired product 7 (4.37 $\mathrm{g}, 70 \%$ ) as a white solid. $\mathrm{R}_{f}$ (cyclohexane/EtOAc: 6/4) 0.28; $[\alpha]_{\mathrm{D}}^{20}-19.0$ (c 0.63, $\left.\mathrm{CHCl}_{3}\right) ;{ }^{1} \mathrm{H} \mathrm{NMR}(300 \mathrm{MHz}$, $\left.\mathrm{CDCl}_{3}\right) \delta 5.46(\mathrm{~s}, 1 \mathrm{H}), 4.36(\mathrm{~s}, 1 \mathrm{H}), 4.17-4.05(\mathrm{~m}, 1 \mathrm{H}), 3.76(\mathrm{~s}, 3 \mathrm{H}), 2.55(\mathrm{~s}, 1 \mathrm{H}), 1.43(\mathrm{~s}, 9 \mathrm{H}), 1.20(\mathrm{~d}, J 6.3 \mathrm{~Hz}$, $3 \mathrm{H}) ;{ }^{13} \mathrm{C}$ NMR $\left(75 \mathrm{MHz}, \mathrm{CDCl}_{3}\right) \delta 171.0,156.1,80.5,68.9,59.0,52.4,28.2,18.8$. Enantio- and diastereoselectivities were determined by HPLC after the asymmetric hydrogenation step on the corresponding benzamide derivative. HPLC : Chiralpak AS-H, hexane/iPrOH 94/6, $1 \mathrm{~mL} / \mathrm{min}, \lambda=215 \mathrm{~nm}$; $\mathrm{t}_{\mathrm{R}}$ $[s y n]=33.8 \mathrm{~min}, \mathrm{t}_{\mathrm{R}}[$ anti $]=37.3 \mathrm{~min}, \mathrm{t}_{\mathrm{R}}[\mathrm{syn}]=41.4 \mathrm{~min}, \mathrm{t}_{\mathrm{R}}[$ anti $]=50.4 \mathrm{~min}$.

Methyl (2R,3R)-2-[(tert-butoxycarbonyl)amino]-3-[(tert-butyldimethylsilyl)oxy]butanoate (8). To a solution of 7 ( $7.23 \mathrm{~g}, 31 \mathrm{mmol}, 1$ equiv) in dry DMF $(28 \mathrm{~mL})$ were added successively imidazole $(0.876 \mathrm{~g}, 12.87 \mathrm{mmol}, 3$ equiv) and $\operatorname{TBSCl}(1.62 \mathrm{~g}, 10.73 \mathrm{mmol}, 2.5$ equiv) at $\mathrm{rt}$. The mixture was stirred for $23 \mathrm{~h}$ at $\mathrm{rt}$, then brine and $\mathrm{Et}_{2} \mathrm{O}$ were added. The aqueous phase was extracted with $\mathrm{Et}_{2} \mathrm{O}$. The combined organic layers were washed with brine, dried over $\mathrm{MgSO}_{4}$, filtered and concentrated. The residue was purified by flash chromatography $\left(\mathrm{SiO}_{2}\right.$, petroleum ether/EtOAc: $96 / 4)$ to give 8 (10.23 g, 95\%) as a colorless oil. $\mathrm{R}_{f}$ (cyclohexane/EtOAc: 95/5) 0.26; $[\alpha]_{\mathrm{D}}^{20}-27.6$ (c 0.78, $\mathrm{CHCl}_{3}$ ); IR (film): 2955, 2935, 2858, 1721, 1498, 1365, 1253, 1168, 834, $778 \mathrm{~cm}^{-1} ;{ }^{1} \mathrm{H} \mathrm{NMR}$ $\left(300 \mathrm{MHz}, \mathrm{CDCl}_{3}\right) \delta 5.26(\mathrm{~d}, J 8.6 \mathrm{~Hz}, 1 \mathrm{H}), 4.23(\mathrm{dd}, J 8.2,3.4 \mathrm{~Hz}, 1 \mathrm{H}), 4.12-4.02(\mathrm{~m}, 1 \mathrm{H}), 3.74(\mathrm{~s}, 3 \mathrm{H}), 1.44(\mathrm{~s}$, 9H), 1.23 (d, J $6.4 \mathrm{~Hz}, 3 \mathrm{H}), 0.86(\mathrm{~s}, 9 \mathrm{H}), 0.04(\mathrm{~s}, 6 \mathrm{H}) ;{ }^{13} \mathrm{C} \mathrm{NMR}\left(75 \mathrm{MHz}, \mathrm{CDCl}_{3}\right) \delta 170.7,155.1,79.7,69.9,59.6$, $51.9,28.3,25.6,20.4,17.9,-4.6,-5.1$.

\section{tert-Butyl (4R,5R)-4-[(tert-butoxycarbonyl)amino]-5-[(tert-butyldimethylsilyl)oxy]-3-oxohexanoate (9)}

To a solution of diisopropylamine (14.1 mL, $100.4 \mathrm{mmol}, 3.6$ equiv) in dry THF (175 mL) was added $n$ butyllithium ( $49 \mathrm{~mL}, 97.7 \mathrm{mmol}, 2 \mathrm{M}, 3.5$ equiv) at $0{ }^{\circ} \mathrm{C}$ and the mixture was stirred at $0{ }^{\circ} \mathrm{C}$ for $45 \mathrm{~min}$. The solution was then cooled to $-45{ }^{\circ} \mathrm{C}$ and tert-butyl acetate $(15 \mathrm{~mL}, 111.6 \mathrm{mmol}, 4$ equiv) was added. The resulting mixture was stirred for $1.5 \mathrm{~h}$ at $-45^{\circ} \mathrm{C}$ before a solution of $8(9.7 \mathrm{~g}, 27.9 \mathrm{mmol}, 1$ equiv) in $50 \mathrm{~mL}$ of dry THF was added via cannula (rinsed with $25 \mathrm{~mL}$ ). The reaction mixture was stirred at $-45{ }^{\circ} \mathrm{C}$ for $22 \mathrm{~h}$. The solution was then warmed to $0{ }^{\circ} \mathrm{C}$ before the reaction was quenched with $200 \mathrm{~mL}$ of saturated $\mathrm{NH}_{4} \mathrm{Cl}$. The aqueous phase was extracted with EtOAc and the combined organic layers were washed with saturated 
$\mathrm{NaHCO}_{3}$ and brine. The organic phase was dried over $\mathrm{MgSO}_{4}$, filtered and concentrated under reduced pressure to afford the crude mixture which was purified by flash column chromatography $\left(\mathrm{SiO}_{2}\right.$, petroleum ether/EtOAc: $92 / 8$ ) to yield the desired compoud 9 (10.5 g, 87\%) as a colorless oil. $\mathrm{R}_{f}$ (cyclohexane/EtOAc: 9/1) 0.49; $[\alpha]_{\mathrm{D}}^{20}-13.2$ (c 2.05, $\mathrm{CHCl}_{3}$ ); IR (film) : 2979, 2931, 2859, 1714, 1500, 1367, 1253, 1161, 833 cm-1. ${ }^{1} \mathrm{H} \mathrm{NMR}$ $\left(300 \mathrm{MHz}, \mathrm{CDCl}_{3}\right.$ ) $\delta 5.22$ (d, J $8.4 \mathrm{~Hz}, 1 \mathrm{H}$ ), 4.29 (dd, J 7.1, $\left.6.4 \mathrm{~Hz}, 1 \mathrm{H}\right), 4.02$ (quint, J $6.2 \mathrm{~Hz}, 1 \mathrm{H}$ ), 3.59 (d, J 16.0 Hz, 1H), 3.45 (d, J 16.1 Hz, 1H), 1.46 (s, 9H), 1.43 (s, 9H), 1.23 (d, J 6.3 Hz, 3H), 0.87 (s, 9H), 0.06 (s, 3H), 0.04 (s, $3 \mathrm{H}) ;{ }^{13} \mathrm{C} \mathrm{NMR}\left(75 \mathrm{MHz}, \mathrm{CDCl}_{3}\right) \delta 201.5,165.9,155.2,82.0,79.9,70.2,64.3,50.4,28.3,27.9,25.7,20.7,17.9$, -4.7, -5.0; HRMS (ESI): calculated for $\mathrm{C}_{21} \mathrm{H}_{41} \mathrm{O}_{6} \mathrm{NSiNa:} 454.2595$, found : 454.2592 .

tert-Butyl (3R,4S,5R)-4-[(tert-butoxycarbonyl)amino]-5-[(tert-butyldimethylsilyl)oxy]-3-hydroxyhexanoate (10) A degassed solution of 9 (100 mg, $0.23 \mathrm{mmol}, 1$ equiv) in EtOH $(3.8 \mathrm{~mL})$ was prepared in a reaction tube and the vessel was purged with argon. Solid catalyst $\left[\{\mathrm{RuCl}((S)-S y n p h o s)\}_{2}(\mu-\mathrm{Cl})_{3}\right]\left[\mathrm{Me}_{2} \mathrm{NH}_{2}\right](3.9 \mathrm{mg}, 0.0023 \mathrm{mmol}$, 0.01 equiv) was then added and the reaction vessel was degassed by three vacuum-argon cycles and then placed under argon in a $250 \mathrm{~mL}$ stainless steel autoclave. The argon atmosphere was replaced with hydrogen by three cycles of pressurizing and the pressure adjusted to 100 bar. The autoclave was heated at $50{ }^{\circ} \mathrm{C}$ and stirring was maintained for $6 \mathrm{~h}$. After cooling, the reaction mixture was concentrated under reduced pressure to afford the crude product which was purified by flash chromatography $\left(\mathrm{SiO}_{2}\right.$, petroleum ether/i $\left.\operatorname{Pr}_{2} \mathrm{O}: 8 / 2\right)$ to afford the desired compound 10 (70 mg, 70\%) as a colorless oil. $R_{f}$ (cyclohexane/EtOAc: $\left.9 / 1\right) 0.41 ;[\alpha]_{D}^{20}-25.0$ (c $\left.0.88, \mathrm{CHCl}_{3}\right) ;{ }^{1} \mathrm{H}$ NMR $\left(300 \mathrm{MHz}, \mathrm{CDCl}_{3}\right) \delta 5.22(\mathrm{~d}, J 9.2 \mathrm{~Hz}, 1 \mathrm{H}), 4.61(\mathrm{dd}, J 4.8,8.5 \mathrm{~Hz}, 1 \mathrm{H}), 4.13(\mathrm{~m}, 1 \mathrm{H}), 3.57$ (bs, $1 \mathrm{H}$ ), 3.31 (dd, J 3.5, $9.3 \mathrm{~Hz}, 1 \mathrm{H}), 2.40$ (dd, J 8.5, 16.0 Hz, 1H), 2.33 (dd, J 4.9, $16.1 \mathrm{~Hz}, 1 \mathrm{H}$ ), $1.46(\mathrm{~s}, 9 \mathrm{H}), 1.44$ (s, 9H), 1.27 (d, J $6.4 \mathrm{~Hz}, 3 \mathrm{H}), 0.88(\mathrm{~s}, 9 \mathrm{H}), 0.08(\mathrm{~s}, 3 \mathrm{H}), 0.08(\mathrm{~s}, 3 \mathrm{H}) ;{ }^{13} \mathrm{C} \mathrm{NMR}\left(75 \mathrm{MHz}, \mathrm{CDCl}_{3}\right) \delta$ 171.1, 155.7, 80.9, 79.3, 71.8, 66.0, 56.9, 40.0, 28.4, 28.1, 25.7, 20.7, 17.8, -4.7, -5.1; HRMS (ESI): calculated for $\mathrm{C}_{21} \mathrm{H}_{43} \mathrm{O}_{6} \mathrm{NSiNa}[\mathrm{M}+\mathrm{Na}]^{+}: 456.2757$, found : 456.2752 .

tert-Butyl (4S,5R)-5-[2-(tert-butoxy)-2-oxoethyl]-4-(R)-1-\{[(tert-butyldimethylsilyl)oxy]ethyl\}-2,2-dimethyloxazolidine-3-carboxylate (11). To a solution of ester 10 (2.6 g, 6 mmol, 1 equiv) in dry $\mathrm{CH}_{2} \mathrm{Cl}_{2}(100 \mathrm{~mL})$ was added distilled dimethoxypropane $(3.9 \mathrm{~mL}, 32 \mathrm{mmol}, 5.3$ equiv) and ( \pm )-camphorsulfonic acid $(0.265 \mathrm{~g}, 1.14$ mmol, 0.19 equiv) at rt. The mixture was stirred at reflux for $1 \mathrm{~h}$. The solution was then cooled to $\mathrm{rt}$ and saturated $\mathrm{NaHCO}_{3}$ was added. The aqueous phase was extracted with $\mathrm{CH}_{2} \mathrm{Cl}_{2}$ and the combined organic layers were washed with saturated $\mathrm{NaHCO}_{3}$ and brine, dried over $\mathrm{MgSO}_{4}$, filtered and concentrated under reduced pressure. The crude product was purified by flash chromatography $\left(\mathrm{SiO}_{2}\right.$, petroleum ether/EtOAc: 93/7 to 8/2 to $6 / 4)$ to afford the desired compound $11(0.757 \mathrm{~g}, 27 \%)$ as a white solid. $\mathrm{R}_{f}$ (cyclohexane/EtOAc: 8/2, ninhydrin) 0.68; $[\alpha]_{\mathrm{D}}^{20}-21.5\left(c 0.79, \mathrm{CHCl}_{3}\right) ;{ }^{1} \mathrm{H} \mathrm{NMR}\left(300 \mathrm{MHz}, \mathrm{C}_{6} \mathrm{D}_{6}\right) \delta 5.00-4.77(\mathrm{~m}, 1 \mathrm{H}), 4.60(\mathrm{~s}, 1 \mathrm{H}), 3.72(\mathrm{~s}$, $1 \mathrm{H}), 2.77-2.05(\mathrm{~m}, 2 \mathrm{H}), 1.59$ (bs, 6H), $1.38(\mathrm{~s}, 9 \mathrm{H}), 1.35(\mathrm{~s}, 9 \mathrm{H}), 1.06(\mathrm{~d}, J 6.4 \mathrm{~Hz}, 3 \mathrm{H}), 0.94(\mathrm{~s}, 9 \mathrm{H}), 0.09(\mathrm{~s}, 3 \mathrm{H})$, $0.03(\mathrm{~s}, 3 \mathrm{H}) ;{ }^{13} \mathrm{C}$ NMR $\left(75 \mathrm{MHz}, \mathrm{C}_{6} \mathrm{D}_{6}\right) \delta 169.2,152.3,79.9,79.4(2 \mathrm{C}), 72.2,67.5,66.5,42.7,28.5,28.2,28.0$, 27.3, 26.2, 21.2, 18.3, -4.4, -4.5; HRMS (ESI): calculated for $\mathrm{C}_{24} \mathrm{H}_{47} \mathrm{O}_{6} \mathrm{NSiNa}[\mathrm{M}+\mathrm{Na}]^{+}: 496.3070$, found : 496.3065.

tert-Butyl (4S,5R)-4-\{(R)-1-[(tert-butyldimethylsilyl)oxy]ethyl\}-2,2-dimethyl-5-(2-oxoethyl)oxazolidine-3-carboxylate (3). Diisobutylaluminum hydride (1 M solution in toluene, $2.3 \mathrm{~mL}, 2.3 \mathrm{mmol}, 1.5$ equiv) was added to a cooled $\left(-85^{\circ} \mathrm{C}\right)$ solution of the ester 11 (733 mg, $1.5 \mathrm{mmol}, 1$ equiv) in $\mathrm{CH}_{2} \mathrm{Cl}_{2}(19 \mathrm{~mL})$. After $1.5 \mathrm{~h}$ at $-85^{\circ} \mathrm{C}$, Rochelle salt was added at $-85{ }^{\circ} \mathrm{C}$ and the resulting mixture was warmed to $\mathrm{rt}$ and stirred overnight. The biphasic solution was extracted with $\mathrm{CH}_{2} \mathrm{Cl}_{2}$. The combined organic extracts were washed with Rochelle salt and brine, dried over $\mathrm{MgSO}_{4}$ and concentrated under reduced pressure. The residue was purified by flash column chromatography (pentane/EtOAc: 9/1) to afford compound $3(1.23 \mathrm{~g}, 77 \%)$ as a colorless oil. R $\mathrm{R}_{f}$ (cyclohexane/EtOAc: 8/2, ninhydrin) 0.41; $[\alpha]_{\mathrm{D}}^{20}-35.4$ (c 0.79, $\mathrm{CHCl}_{3}$ ); IR (film): 3439, 2978, 2937, 2856, 1697, 1463, 1376, 1257, 1079, 838, 777, $741 \mathrm{~cm}^{-1}$; ${ }^{1} \mathrm{H}$ NMR (300 MHz, C $\left.6 \mathrm{D}_{6}\right) \delta 9.44$ (dd, J 2.9, $1.5 \mathrm{~Hz}, 1 \mathrm{H}$ ), 4.76 (ddd, J 
8.4, 5.6, $4.3 \mathrm{~Hz}, 1 \mathrm{H}$ ), 4.70 (bs, 1H), 3.50 (bs, 1H), 2.27 (ddd, J 16.0, 8.4, $2.9 \mathrm{~Hz}, 1 \mathrm{H}$ ), 2.13 (ddd, J 16.0, $4.3,1.5$ $\mathrm{Hz}, 1 \mathrm{H}), 1.58$ (bs, 6H), 1.41 (s, 9H), 0.96 (s, 9H), 0.95 (bs, 3H), 0.10 (s, 3H), 0.05 (s, 3H); ${ }^{13} \mathrm{C} \mathrm{NMR}\left(75 \mathrm{MHz}, \mathrm{C}_{6} \mathrm{D}_{6}\right)$ $\delta 198.5,152.2,94.5,79.5,69.8,67.6,65.4,49.6,28.3,27.2,27.1,26.0,20.9,18.1,-4.3,-4.7$; HRMS (ESI): calculated for $\mathrm{C}_{20} \mathrm{H}_{39} \mathrm{O}_{5} \mathrm{NSiNa}[\mathrm{M}+\mathrm{Na}]^{+}: 424.2495$, found : 424.2490.

tert-Butyl (4S,5R)-4-\{(R)-1-[(tert-butyldimethylsilyl)oxy]ethyl\}-5-((R)-2-hydroxybut-3-en-1-yl)-2,2-dimethyloxazolidine-3-carboxylate (12). A $0.7 \mathrm{M}$ solution of vinyl magnesium bromide in THF $(4.8 \mathrm{~mL}, 3.33 \mathrm{mmol}, 3$ equiv) was added to a solution of aldehyde 3 (447 mg, $1.11 \mathrm{mmol}, 1$ equiv) in dry THF (1.9 mL) at $-78^{\circ} \mathrm{C}$. The mixture was stirred at $-78{ }^{\circ} \mathrm{C}$ for $4 \mathrm{~h}$. Saturated $\mathrm{NH}_{4} \mathrm{Cl}$ was added at $-78{ }^{\circ} \mathrm{C}$ and the solution was warmed to rt. The aqueous phase was extracted with EtOAc and the combined organic layers were washed with saturated $\mathrm{NH}_{4} \mathrm{Cl}$, dried over $\mathrm{MgSO}_{4}$, filtered and concentrated under vacuum. Purification of the crude material by flash chromatography $\left(\mathrm{SiO}_{2}\right.$, pentane/i $\mathrm{Pr}_{2} \mathrm{O}:$ 8/2 to pentane/EtOAc: 8/2) afforded 12 (major diastereomer, $231 \mathrm{mg}$, $48 \%)$ as well as the minor diastereoisomer $(137 \mathrm{mg}, 29 \%)$ as colorless oils. $\mathrm{R}_{f}$ (cyclohexane/EtOAc: $8 / 2$, ninhydrin) 0.46 (minor), 0.37 (major); $[\alpha]_{\mathrm{D}}^{20}$ (major): -35.8 (c 0.74, $\mathrm{CHCl}_{3}$ ); ${ }^{1} \mathrm{H} \mathrm{NMR}$ (major) $\left(300 \mathrm{MHz}^{\mathrm{CDCl}}\right)_{3} \delta$ 5.82 (ddd, $1 \mathrm{H}, J$ 17.2, 10.4, $5.6 \mathrm{~Hz}$ ), 5.35 (dd, 1H, J 17.2, $1.7 \mathrm{~Hz}), 5.06-4.99(\mathrm{~m}, 1 \mathrm{H}), 4.72$ (s I, $1 \mathrm{H}), 4.56$ (ddd, $1 \mathrm{H}, J$ 9.9, 5.5, $3.3 \mathrm{~Hz}), 4.38(\mathrm{~m}, 1 \mathrm{H}), 3.60$ (bs, 1H), $2.69(\mathrm{~s}, 1 \mathrm{H}), 2.02-1.48(\mathrm{~m}, 8 \mathrm{H}), 1.41(\mathrm{~s}, 9 \mathrm{H}), 1.01(\mathrm{~d}, 3 \mathrm{H}, J$ $5.2 \mathrm{~Hz}$ ), $0.96(\mathrm{~s}, 9 \mathrm{H}), 0.11(\mathrm{~s}, 3 \mathrm{H}), 0.06(\mathrm{~s}, 3 \mathrm{H}) ;{ }^{13} \mathrm{C}$ NMR (major) (75 MHz, $\left.\mathrm{C}_{6} \mathrm{D}_{6}\right) \delta$ 150.2, 141.2, 114.1, 94.4, 79.4, 74.1, 71.4, 68.3, 65.4, 43.9, 28.3, 27.4, 27.1, 26.0, 21.0, 18.1, -4.3, -4.7; HRMS (ESI): calculated for $\mathrm{C}_{22} \mathrm{H}_{43} \mathrm{O}_{5} \mathrm{NSiNa}[\mathrm{M}+\mathrm{Na}]^{+}: 452.2808$, found : 452.2803 .

tert-Butyl (4S,5R)-5-((R)-2-((tert-butyldimethylsilyl)oxy)but-3-en-1-yl)-4-((R)-1-((tert-butyldimethylsilyl)oxy) ethyl)-2,2-dimethyloxazolidine-3-carboxylate (2). To a solution of 12 (146 mg, $0.34 \mathrm{mmol}, 1$ equiv) in dry DMF $(1 \mathrm{~mL}$ ) were added successively TBSCl ( $75 \mathrm{mg}, 0.5 \mathrm{mmol}, 1.5$ equiv) and imidazole ( $46 \mathrm{mg}, 0.68 \mathrm{mmol}, 2$ equiv) at $0{ }^{\circ} \mathrm{C}$. The mixture was stirred for $19 \mathrm{~h}$ at $\mathrm{rt}$, then brine and EtOAc were added. The aqueous phase was extracted with EtOAc. The combined organic layers were washed with brine, dried over $\mathrm{MgSO}_{4}$, filtered and concentrated. The residue was purified by flash chromatography $\left(\mathrm{SiO}_{2}\right.$, petroleum ether/i $\left.\operatorname{Pr}_{2} \mathrm{O}: 95 / 5\right)$ to give 2 (146 mg, 79\%) as a colorless oil. $\mathrm{R}_{f}$ (cyclohexane/EtOAc: 85/15, ninhydrin) 0.79; $[\alpha]_{\mathrm{D}}^{20}-11.7\left(c 0.74, \mathrm{CHCl}_{3}\right) ; \mathrm{IR}$ (film): 2957, 2930, 2858, 1695, 1471, 1464, 1364, 1256, 1082, 836, $776 \mathrm{~cm}^{-1} ;{ }^{1} \mathrm{H}$ NMR $\left(300 \mathrm{MHz}, \mathrm{C}_{6} \mathrm{D}_{6}\right) \delta 5.95$ (ddd, J 17.2, 10.3, 6.8 Hz, 1H), 5.29 (d, J $17.1 \mathrm{~Hz}, 1 \mathrm{H}), 5.05(\mathrm{~d}, J 10.3 \mathrm{~Hz}, 1 \mathrm{H}), 4.90-4.21(\mathrm{~m}, 3 \mathrm{H}), 3.63(\mathrm{bs}, 1 \mathrm{H})$, $2.17-1.54(\mathrm{~m}, 8 \mathrm{H}), 1.43(\mathrm{~s}, 9 \mathrm{H}), 1.01$ (bs, 12H), $0.96(\mathrm{~s}, 9 \mathrm{H}), 0.14(\mathrm{~s}, 3 \mathrm{H}), 0.12(\mathrm{~s}, 3 \mathrm{H}), 0.11(\mathrm{~s}, 3 \mathrm{H}), 0.06(\mathrm{~s}, 3 \mathrm{H})$; ${ }^{13} \mathrm{C}$ NMR $\left(75 \mathrm{MHz}, \mathrm{C}_{6} \mathrm{D}_{6}\right) \delta 152.2,141.2,114.8,93.8,79.2,72.1,71.2,68.1,65.4,45.4,28.3,27.4,27.1,26.1$, 26.0, 21.1, 18.3, 18.1, $-4.2,-4.6(2 \mathrm{C}),-4.7$; HRMS (ESI): calculated for $\mathrm{C}_{28} \mathrm{H}_{57} \mathrm{O}_{5} \mathrm{NSi}{ }_{2} \mathrm{Na}[\mathrm{M}+\mathrm{Na}]^{+}: 566.3673$, found : 566.3668 .

tert-Butyl $\quad(4 S, 5 R)-5-\{(S)-2-[($ tert-butyldimethylsilyl)oxy]-4-hydroxybutyl\}-4-\{(R)-1-[(tert-butyldimethylsilyl)oxy]ethyl\}-2,2-dimethyloxazolidine-3-carboxylate (13). To a solution of $\mathbf{2}$ (137 $\mathrm{mg}, 0.25 \mathrm{mmol}, 1$ equiv) in dry THF (1.4 mL) was added $\mathrm{BH}_{3} . \mathrm{Me}_{2} \mathrm{~S}\left(2 \mathrm{M}\right.$ in THF, $0.25 \mathrm{~mL}, 0.75 \mathrm{mmol}, 3$ equiv) at $0{ }^{\circ} \mathrm{C}$. The mixture was stirred at $0{ }^{\circ} \mathrm{C}$ for $1 \mathrm{~h}$ then allowed to rise to rt. $\mathrm{NaOH} 3 \mathrm{M}\left(1.25 \mathrm{~mL}, 3.75 \mathrm{mmol}, 15\right.$ equiv) and $35 \% \mathrm{H}_{2} \mathrm{O}_{2}$ in $\mathrm{H}_{2} \mathrm{O}(0.32 \mathrm{~mL}$, $3.75 \mathrm{mmol}, 15$ equiv) were added at $0{ }^{\circ} \mathrm{C}$ and the resulting solution was stirred at rt for $1 \mathrm{~h}$. Saturated $\mathrm{NH}_{4} \mathrm{Cl}$ was added and the aqueous phase was extracted with $\mathrm{CH}_{2} \mathrm{Cl}_{2}$. The combined organic layers were washed with saturated $\mathrm{NH}_{4} \mathrm{Cl}$, dried over $\mathrm{MgSO}_{4}$, filtered and concentrated under reduced pressure. The residue was purified by flash chromatography ( $\mathrm{SiO}_{2}$, petroleum ether/EtOAc: 9/1 to 8/2) to give 13 (105 mg, 75\%) as a pale yellow oil. $\mathrm{R}_{f}$ (cyclohexane/EtOAc: $8 / 2$, ninhydrin) $0.41 ;{ }^{1} \mathrm{H} \mathrm{NMR}\left(400 \mathrm{MHz}, \mathrm{C}_{6} \mathrm{D}_{6}, 50{ }^{\circ} \mathrm{C}\right) \delta 4.74-4.60(\mathrm{~m}, 1 \mathrm{H})$, 4.52 (ddd, J 9.8, 5.7, 3.0 Hz, 1H), $4.27(\mathrm{~m}, 1 \mathrm{H}), 3.82-3.63(\mathrm{~m}, 2 \mathrm{H}), 3.58(\mathrm{dd}, J 5.7,3.1 \mathrm{~Hz}, 1 \mathrm{H}), 2.04-1.85(\mathrm{~m}$, $2 \mathrm{H}), 1.82-1.67(\mathrm{~m}, 2 \mathrm{H}), 1.62$ (bs, 3H), $1.44(\mathrm{~s}, 9 \mathrm{H}), 1.43$ (bs, 3H), 1.11 (d, J $6.5 \mathrm{~Hz}, 3 \mathrm{H}), 0.97$ (s, $9 \mathrm{H}), 0.97$ (s, $9 \mathrm{H}), 0.13(\mathrm{~s}, 3 \mathrm{H}), 0.13(\mathrm{~s}, 3 \mathrm{H}), 0.12(\mathrm{~s}, 3 \mathrm{H}), 0.09(\mathrm{~s}, 3 \mathrm{H}) ;{ }^{13} \mathrm{C} \mathrm{NMR}\left(100 \mathrm{MHz}, \mathrm{C}_{6} \mathrm{D}_{6}, 50{ }^{\circ} \mathrm{C}\right) \delta$ 152.6, 94.7, 79.6, 
72.3, 69.2, 69.0, 66.6, 59.8, 44.9, 38.9, 28.6, 27.7, 27.3, 26.3, 26.2, 21.4, 18.3, 18.3, -3.9, -4.1, -4.4, -4.5; HRMS (ESI): calculated for $\mathrm{C}_{28} \mathrm{H}_{59} \mathrm{O}_{6} \mathrm{NSi}{ }_{2} \mathrm{Na}[\mathrm{M}+\mathrm{Na}]^{+}: 584.3779$, found : 584.3773 .

tert-Butyl $\quad(4 S, 5 R)-5-\{(R)-2-[($ tert-butyldimethylsilyl)oxy]-4-\{[(1-phenyl-1H-tetrazol-5-yl)thio]butyl\}-4-\{[(R)-1(tert-butyldimethylsilyl)oxy]ethyl\}-2,2-dimethyloxazolidine-3-carboxylate (14). To a solution of 13 (107 mg, $0.19 \mathrm{mmol}, 1$ equiv) in dry THF $(1.6 \mathrm{~mL})$ were successively added triphenylphosphine $(60 \mathrm{mg}, 0.23 \mathrm{mmol}, 1.2$ equiv), 1-phenyl-1H-tetrazole-5-thiol ( $41 \mathrm{mg}, 0.23 \mathrm{mmol}, 1.2$ equiv) and DIAD (0.12 mL, $0.23 \mathrm{mmol}, 1.2 \mathrm{equiv}$ ) dropwise at $\mathrm{rt}$. The mixture was stirred at $\mathrm{rt}$ for $22.5 \mathrm{~h}$. Saturated $\mathrm{NaHCO}_{3}$ was added and the aqueous phase was extracted with EtOAc. The combined organic phases were washed with saturated $\mathrm{NaHCO}_{3}$, dried over $\mathrm{MgSO}_{4}$, filtered and concentrated under reduced pressure. The residue was purified by flash chromatography $\left(\mathrm{SiO}_{2}\right.$, petroleum ether/iPr $2 \mathrm{O}: 9 / 1$ to petroleum ether/EtOAc: 9/1) to give 14 (109 $\left.\mathrm{mg}, 80 \%\right)$ as a pale yellow oil. $\mathrm{R}_{f}$ (cyclohexane/EtOAc: 8/2, ninhydrin, UV) 0.63; ${ }^{1} \mathrm{H} \mathrm{NMR}\left(400 \mathrm{MHz}, \mathrm{CDCl}_{3}, 50{ }^{\circ} \mathrm{C}\right) \delta 7.62-7.48(\mathrm{~m}, 5 \mathrm{H})$, 4.46 (bs, 1H), 4.33 (ddd, J 10.0, 5.7, $2.9 \mathrm{~Hz}, 1 \mathrm{H}), 4.15-4.06(\mathrm{~m}, 1 \mathrm{H}), 3.58-3.40(\mathrm{~m}, 3 \mathrm{H}), 2.24-2.13(\mathrm{~m}, 1 \mathrm{H})$, $2.06-1.86(\mathrm{~m}, 2 \mathrm{H}), 1.84-1.74(\mathrm{~m}, 1 \mathrm{H}), 1.56(\mathrm{~s}, 3 \mathrm{H}), 1.49(\mathrm{~s}, 9 \mathrm{H}), 1.46(\mathrm{~s}, 3 \mathrm{H}), 1.11(\mathrm{~d}, J 6.5 \mathrm{~Hz}, 3 \mathrm{H}), 0.91(\mathrm{~s}$, 9H), 0.89 (s, 9H), 0.09 (s, 6H), 0.07 (s, 3H), $0.04(\mathrm{~s}, 3 \mathrm{H}) ;{ }^{13} \mathrm{C} \mathrm{NMR}\left(100 \mathrm{MHz}, \mathrm{CDCl}_{3}, 50{ }^{\circ} \mathrm{C}\right) \delta 154.6,152.7,134.4$, $130.1,129.9,124.2,94.6,80.1,71.7,69.0,68.7,66.3,44.2,35.8,29.9,28.7,27.4,27.2,26.2,26.1,21.4,18.2$ (2C), $-4.0,-4.1,-4.3(2 \mathrm{C})$; HRMS (ESI): calculated for $\mathrm{C}_{35} \mathrm{H}_{63} \mathrm{O}_{5} \mathrm{~N}_{5} \mathrm{Si}_{2} \mathrm{SNa}[\mathrm{M}+\mathrm{Na}]^{+}:$744.3986, found : 744.3981. tert-Butyl $\quad(4 S, 5 R)-5-\{(R)-2[($ tert-butyldimethylsilyl)oxy]-4-[(1-phenyl-1H-tetrazol-5-yl)sulfonyl]butyl\}-4-\{(R)1-[(tert-butyldimethylsilyl)oxy]ethyl\}-2,2-dimethyloxazolidine-3-carboxylate $\left(A^{\prime}\right)$. To a solution of 14 (95 mg, $0.13 \mathrm{mmol}, 1$ equiv) in dry $\mathrm{CH}_{2} \mathrm{Cl}_{2}(2 \mathrm{~mL})$ were added $\mathrm{NaHCO}_{3}(33 \mathrm{mg}, 0.39 \mathrm{mmol}, 3$ equiv) and $\mathrm{m}$-CPBA (67 mg, 0.39 mmol, 3 equiv) at $0{ }^{\circ} \mathrm{C}$. The mixture was stirred for $14 \mathrm{~h}$ at rt. Saturated $\mathrm{Na}_{2} \mathrm{~S}_{2} \mathrm{O}_{3}$ was added at $0{ }^{\circ} \mathrm{C}$ and the mixture was diluted with $\mathrm{CH}_{2} \mathrm{Cl}_{2}$. The white solid was filtered and the aqueous layer was extracted with $\mathrm{CH}_{2} \mathrm{Cl}_{2}$. The combined organic phases were dried over $\mathrm{MgSO}_{4}$, filtered and concentrated under reduced pressure. The residue was purified by flash chromatography $\left(\mathrm{SiO}_{2}\right.$, pentane $/ \mathrm{iPr}_{2} \mathrm{O}: 9 / 1$ to $\left.8 / 2\right)$ to give $\mathrm{A}^{\prime}(67$ $\mathrm{mg}, 68 \%$ ) as a pale yellow oil. $\mathrm{R}_{f}$ (cyclohexane/EtOAc: $8 / 2$, ninhydrin, UV) $0.58 ;{ }^{1} \mathrm{H} \mathrm{NMR}\left(300 \mathrm{MHz}^{\mathrm{CDCl}}{ }_{3}\right) \delta$ 7.74-7.65 (m, 2H), $7.65-7.55(\mathrm{~m}, 3 \mathrm{H}), 4.54(\mathrm{bs}, 1 \mathrm{H}), 4.41-4.24(\mathrm{~m}, 1 \mathrm{H}), 4.18-4.05(\mathrm{~m}, 1 \mathrm{H}), 3.94-3.74(\mathrm{~m}$, $2 \mathrm{H}), 3.43(\mathrm{bs}, 1 \mathrm{H}), 2.36-2.21(\mathrm{~m}, 1 \mathrm{H}), 2.19-2.05(\mathrm{~m}, 1 \mathrm{H}), 1.80-1.60(\mathrm{~m}, 2 \mathrm{H}), 1.55(\mathrm{bs}, 3 \mathrm{H}), 1.47(\mathrm{~s}, 9 \mathrm{H}), 1.44$ (bs, 3H), 1.08 (d, J $6.6 \mathrm{~Hz}, 3 \mathrm{H}), 0.89(\mathrm{~s}, 9 \mathrm{H}), 0.86(\mathrm{~s}, 9 \mathrm{H}), 0.09(\mathrm{~s}, 3 \mathrm{H}), 0.07(\mathrm{~s}, 3 \mathrm{H}), 0.05(\mathrm{~s}, 3 \mathrm{H}), 0.01(\mathrm{~s}, 3 \mathrm{H}) ;{ }^{13} \mathrm{C}$ $\operatorname{NMR}\left(75 \mathrm{MHz}_{\mathrm{CDCl}}\right) \delta$ 153.7, 152.7, 133.3, 131.6, 129.9, 125.2, 94.4, 80.2, 70.3, 68.1, 67.7, 64.7, 52.8, 43.4, 30.3, 28.6, 27.0 (2C), 26.1, 26.0, 21.2, 18.1, 18.1, -4.1, -4.3, -4.6 (2C); HRMS (ESI): calculated for $\mathrm{C}_{35} \mathrm{H}_{63} \mathrm{O}_{7} \mathrm{~N}_{5} \mathrm{Si}_{2} \mathrm{SNa}[\mathrm{M}+\mathrm{Na}]^{+}:$776.3884, found : 776.3879 .

$(2 S, 3 S)(E)-2-[($ tert-Butyldimethylsilyl)oxy]-5-iodo-3-methylhex-4-en-1-ol (15). To a solution of $(2 S, 3 S, E)-2-$ ((tert-butyldimethylsilyl)oxy)-5-iodo-3-methylhex-4-en-1-ol ${ }^{19}$ (150 mg, $0.31 \mathrm{mmol}, 1$ equiv) in EtOH (70 mL) was added PPTS $(7.8 \mathrm{mg}, 0.03 \mathrm{mmol}, 0.1$ equiv) at $\mathrm{rt}$. After $24 \mathrm{~h}$ at $\mathrm{rt}$, the mixture was concentrated under reduced pressure. Purification by flash chromatography on silica gel (petroleum ether/Et ${ }_{2} \mathrm{O}=100: 0$ to 80:20) afforded the alcohol 15 as a pale yellow oil (55 mg, 47\%) and bis-protected alcohol was recovered (57 mg, 38\%). $[\alpha]_{\mathrm{D}}^{20}-35.5$ (c 0.64, $\mathrm{CHCl}_{3}$ ); IR (neat): 3416, 1636, 1471, 1462, 1378, 1361, 1525, 1150, 1098, 1046,1006 $\mathrm{cm}^{-1} ;{ }^{1} \mathrm{H}$ NMR $\left(400 \mathrm{MHz}, \mathrm{CDCl}_{3}\right) \delta 6.01(\mathrm{dq}, J 10.2,1.5 \mathrm{~Hz}, 1 \mathrm{H}), 3.61-3.48(\mathrm{~m}, 3 \mathrm{H}), 2.69(\mathrm{~m}, 1 \mathrm{H}), 2.38(\mathrm{~d}, J 1.5$ $\mathrm{Hz}, 3 \mathrm{H}), 1.71$ (brt, J $6.0 \mathrm{~Hz}, 1 \mathrm{H}), 0.97$ (d, J $6.9 \mathrm{~Hz}, 3 \mathrm{H}), 0.90(\mathrm{~s}, 9 \mathrm{H}), 0.10(\mathrm{~s}, 3 \mathrm{H}), 0.08(\mathrm{~s}, 3 \mathrm{H}) ;{ }^{13} \mathrm{C} \mathrm{NMR}(100 \mathrm{MHz}$ $\left.\mathrm{CDCl}_{3}\right) \delta 143.5,94.5,75.8,64.3,38.3,27.9,25.9,18.0$ (3C), 16.5, -4.4, -4.5; MS (EI) m/z: 339 (4), 313 (9, [M$\left.t \mathrm{Bu}]^{+}\right), 255$ (20), 195 (37), 186 (5), 185 (11), 181 (10), 175 (11), 119 (5), 117 (14), 115 (12), 111 (7), 105 (30), 103 (23), 94 (9), 93 (9), 77 (11), 75 (98), 73 (100).

tert-Butyl $\quad(4 S, 5 R)-5-\{(2 S, 4 E, 6 R, 7 S, 8 E)-2,6$-bis[(tert-butyldimethylsilyl)oxy]-9-iodo-7-methyldeca-4,8-dien-1yl\}-4-\{(R)-1-[(tert-butyldimethylsilyl)oxy]ethyl\}-2,2-dimethyloxazolidine-3-carboxylate (16). To a solution of 15 (40 mg, $0.11 \mathrm{mmol}, 1$ equiv) in dry $\mathrm{CH}_{2} \mathrm{Cl}_{2}$ were added at rt MS $4 \AA$ (30 mg), TPAP (3 mg, $0.01 \mathrm{mmol}, 0.08$ 
equiv) and NMO (15 mg, $0.13 \mathrm{mmol}, 1.2$ equiv). The mixture was stirred for $2 \mathrm{~h}$ at $\mathrm{rt}$ and filtered over a pad of celite $\left(\mathrm{CH}_{2} \mathrm{Cl}_{2}\right)$. The crude aldehyde was used directly into the next step. To a solution of $\mathbf{A}$ (55 $\mathrm{mg}, 0.07 \mathrm{mmol}$, 1 equiv) in dry THF (1.5 mL) was added dropwise KHMDS ( $0.15 \mathrm{~mL}, 0.07 \mathrm{mmol}, 0.5 \mathrm{M}$ in toluene, 1.05 equiv) at $-78{ }^{\circ} \mathrm{C}$. The brown solution was stirred for $30 \mathrm{~min}$ at $-78{ }^{\circ} \mathrm{C}$ before adding dropwise a solution of the crude aldehyde (40 mg, $0.11 \mathrm{mmol}, 1.5$ equiv) in THF $(0.5 \mathrm{~mL})$. The mixture was stirred $2 \mathrm{~h}$ at $-78{ }^{\circ} \mathrm{C}$ then $1 \mathrm{~h}$ at $\mathrm{rt}$. An aqueous solution of $\mathrm{NH}_{4} \mathrm{Cl}$ was added and the phases were separated. The aqueous layer was extracted twice with EtOAc. The combined organic layers were washed with water, dried over $\mathrm{Na}_{2} \mathrm{SO}_{4}$ and concentrated under reduced pressure. Purification by flash chromatography on silica gel $\left(\mathrm{SiO}_{2}\right.$, pentane/Et ${ }_{2} \mathrm{O}: 100 / 0$ to $\left.95 / 5\right)$ afforded 16 as a colorless oil (50 mg, 76\%, $E / Z>95: 5$, mixture of rotamers). $[\alpha]_{\mathrm{D}}^{20}-33.0$ (c $\left.0.78, \mathrm{CHCl}_{3}\right) ; \mathrm{IR}$ (neat): 1692, 1462, 1387, 1365, 1253, 1178, 1079, $1025 \mathrm{~cm}^{-1} ;{ }^{1} \mathrm{H} N M R\left(400 \mathrm{MHz}, \mathrm{C}_{6} \mathrm{D}_{6}\right) \delta 6.18$ (dq, J $10.0,1.5$ $\mathrm{Hz}, 1 \mathrm{H}), 5.84(\mathrm{~m}, 1 \mathrm{H}), 5.54$ (ddt, J 15.9, 6.5, $1.1 \mathrm{~Hz}), 4.70$ (bs, $1 \mathrm{H}), 4.56$ (ddd, J 9.3, 5.9, 3.6 Hz, $1 \mathrm{H}), 4.18(\mathrm{~m}$, $1 \mathrm{H}), 3.90(\mathrm{t}, J 6.5 \mathrm{~Hz}, 1 \mathrm{H}), 3.64(\mathrm{dd}, J 5.9,2.7 \mathrm{~Hz}, 1 \mathrm{H}), 2.54(\mathrm{~m}, 1 \mathrm{H}), 2.45(\mathrm{~m}, 1 \mathrm{H}), 2.37(\mathrm{~m}, 1 \mathrm{H}), 2.27(\mathrm{~d}, J 1.5 \mathrm{~Hz}$, $3 \mathrm{H}), 2.01-1.86(\mathrm{~m}, 2 \mathrm{H}), 1.73(\mathrm{~s}, 3 \mathrm{H}), 1.64(\mathrm{~s}, 3 \mathrm{H}), 1.45(\mathrm{~s}, 9 \mathrm{H}), 1.15(\mathrm{~d}, J 6.6 \mathrm{~Hz}, 3 \mathrm{H}), 1.01(\mathrm{~s}, 9 \mathrm{H}), 1.00(\mathrm{~s}, 18 \mathrm{H})$, $0.90(\mathrm{~d}, \mathrm{~J} 6.9 \mathrm{~Hz}, 3 \mathrm{H}), 0.16-0.14(\mathrm{~m}, 9 \mathrm{H}), 0.12-0.09(\mathrm{~m}, 9 \mathrm{H}) ;{ }^{13} \mathrm{C} \mathrm{NMR}\left(100 \mathrm{MHz}, \mathrm{C}_{6} \mathrm{D}_{6}\right) \delta 152.4,144.4,134.3$, 128.3, 94.5, 93.7, 79.4, 77.2, 72.1, 70.3, 68.8, 66.4, 43.8, 43.1, 39.4, 28.5 (3C), 27.9 (2C), 27.5, 26.23 (3C), 26.16 (3C), 26.12 (3C), 21.4, 18.30, 18.25, 18.23, 16.0, -3.9, -4.0, -4.26, -4.29, -4.5 (2C); HRMS (ESI): calculated for $\mathrm{C}_{41} \mathrm{H}_{82} \mathrm{INO}_{6} \mathrm{Si}_{3} \mathrm{Na}[\mathrm{M}+\mathrm{Na}]^{+}:$918.4387, found : 918.4382 .

2-[(4-Methoxybenzyl)oxy]ethan-1-ol (21). To a slurry of $\mathrm{NaH}$ (60\% dispersion in oil, $7.0 \mathrm{~g}, 110 \mathrm{mmol}, 1$ equiv) in dry THF $(300 \mathrm{~mL})$ were added dropwise ethylene glycol 20 (28.8 $\mathrm{mL}, 510 \mathrm{mmol}, 3$ equiv), tetrabutylammonium iodide $(7.1 \mathrm{~g}, 17 \mathrm{mmol}, 0.1$ equiv) and para-methoxylbenzyl chloride $(23.2 \mathrm{~mL}, 9.8$ mmol, 1 equiv) at $0{ }^{\circ} \mathrm{C}$. The mixture was stirred for $4 \mathrm{~h}$ under reflux and a saturated aqueous ammonium chloride solution was added. The aqueous layer was extracted with EtOAc and the combined organic layers were washed with brine, dried, filtered and concentrated. Flash chromatography ( $\mathrm{SiO}_{2}$, petroleum ether/EtOAc: 55/45) gave the alcohol 21 as a yellow oil (27.45 g, 88\%). $\mathrm{R}_{f}$ (cyclohexane/EtOAc:6/4, $\mathrm{KMnO}_{4}, \mathrm{UV}$ ) 0.29; ${ }^{1} \mathrm{H}$ NMR $\left(300 \mathrm{MHz}, \mathrm{CDCl}_{3}\right) \delta 7.28(\mathrm{~d}, J 8.6 \mathrm{~Hz}, 2 \mathrm{H}), 6.89(\mathrm{~d}, J 8.6 \mathrm{~Hz}, 2 \mathrm{H}), 4.49(\mathrm{~s}, 2 \mathrm{H}), 3.81(\mathrm{~s}, 3 \mathrm{H}), 3.78-$ $3.66(\mathrm{~m}, 2 \mathrm{H}), 3.56(\mathrm{t}, J 4.9 \mathrm{~Hz}, 2 \mathrm{H}), 2.57(\mathrm{t}, J 6.1 \mathrm{~Hz}, 1 \mathrm{H}) ;{ }^{13} \mathrm{C} \mathrm{NMR}\left(75 \mathrm{MHz}, \mathrm{CDCl}_{3}\right) \delta$ 159.2, 129.9, 129.3, 113.7, $72.8,71.0,61.7,55.1$.

2-[(4-Methoxybenzyl)oxy\}acetaldehyde (18). Dess-Martin periodinane (2.54 g, $6 \mathrm{mmol}, 1.1$ equiv) was added to a solution of alcohol $21\left(1 \mathrm{~g}, 5.5 \mathrm{mmol}, 1\right.$ equiv) in dry $\mathrm{CH}_{2} \mathrm{Cl}_{2}(30 \mathrm{~mL})$ at $0{ }^{\circ} \mathrm{C}$. After stirring $3 \mathrm{~h}$ at $\mathrm{rt}$, the reaction was quenched with saturated $\mathrm{NaHCO}_{3}$ and the mixture was stirred vigorously until two clear layers were obtained. The layers were separated and the aqueous layer was extracted with EtOAc. The combined organic layers were dried over $\mathrm{MgSO}_{4}$ and the solvent was removed under reduced pressure to a crude mixture which was purified by flash chromatography $\left(\mathrm{SiO}_{2}\right.$, petroleum ether/EtOAc: 6/4) to give the alcohol 18 as a yellow oil (0.605 g, 61\%). $\mathrm{R}_{f}$ (cyclohexane/EtOAc:4/6, $\left.\mathrm{KMnO}_{4}, \mathrm{UV}\right) 0.59 ;{ }^{1} \mathrm{H} \mathrm{NMR}\left(300 \mathrm{MHz}, \mathrm{CDCl}_{3}\right) \delta 9.69$ (s, $1 \mathrm{H}), 7.28(\mathrm{~d}, J 8.7 \mathrm{~Hz}, 2 \mathrm{H}), 6.89(\mathrm{~d}, J 8.7 \mathrm{~Hz}, 2 \mathrm{H}), 4.55(\mathrm{~s}, 2 \mathrm{H}), 4.06(\mathrm{~d}, J 0.7 \mathrm{~Hz}, 2 \mathrm{H}), 3.80(\mathrm{~s}, 3 \mathrm{H}) ;{ }^{13} \mathrm{C} \mathrm{NMR}(75$ $\left.\mathrm{MHz}_{2} \mathrm{CDCl}_{3}\right) \delta 200.6,159.6,129.7,128.8,113.9,74.9,73.2,55.2$.

Methyl (tert-butoxycarbonyl)glycinate (19). Glycine methyl ester hydrochloride 22 (7.28 g, 58 mmol, 1 equiv) was suspended in $\mathrm{CH}_{2} \mathrm{Cl}_{2}(200 \mathrm{~mL})$ and $\mathrm{Et}_{3} \mathrm{~N}\left(9 \mathrm{~mL}, 64 \mathrm{mmol}, 1.1\right.$ equiv) was added at $0{ }^{\circ} \mathrm{C}$. The mixture was stirred for $30 \mathrm{~min}$ at $0^{\circ} \mathrm{C}$ and $\mathrm{Boc}_{2} \mathrm{O}$ (13.95 g, $64 \mathrm{mmol}, 1.1$ equiv) was added at rt. The reaction was stirred for $26 \mathrm{~h}$ at $\mathrm{rt}$ and $\mathrm{H}_{2} \mathrm{O}(150 \mathrm{~mL})$ was added. The mixture was extracted with $\mathrm{CH}_{2} \mathrm{Cl}_{2}$ and the organic phase was washed with $100 \mathrm{~mL}$ of $\mathrm{HCl}(1 \mathrm{~N}), 100 \mathrm{~mL}$ of saturated $\mathrm{Na}_{2} \mathrm{CO}_{3}$ and $100 \mathrm{~mL}$ of brine. The organic layer was dried over $\mathrm{MgSO}_{4}$, filtered, and the solvent was removed under reduced pressure. Flash chromatography of the crude mixture $\left(\mathrm{SiO}_{2}\right.$, petroleum ether/EtOAc: $\left.7 / 3\right)$ gave the desired compound 19 as a colorless oil (10.59 
g, 97\%). $\mathrm{R}_{f}$ (cyclohexane/EtOAc:1/1, $\mathrm{KMnO}_{4}$ ) 0.63; ${ }^{1} \mathrm{H}$ NMR (300 MHz, $\left.\mathrm{CDCl}_{3}\right) \delta 5.00$ (bs, $\left.1 \mathrm{H}\right), 3.92(\mathrm{~d}, J 5.5 \mathrm{~Hz}$, $2 \mathrm{H}), 3.75$ (s, 3H), 1.45 (s, 9H); ${ }^{13} \mathrm{C} N M R\left(75 \mathrm{MHz} \mathrm{CDCl}_{3}\right) \delta 170.8,155.7,80.0,52.2,42.2,28.3$.

Methyl 2-[(tert-butoxycarbonyl)amino]-4-[(4-methoxybenzyl)oxy]-3-oxobutanoate (17). To a solution of diisopropylamine $\left(11.6 \mathrm{~mL}, 82.5 \mathrm{mmol}, 3.3\right.$ equiv) in THF $(90 \mathrm{~mL})$, was added dropwise at $-78{ }^{\circ} \mathrm{C}$, a $2.3 \mathrm{M}$ solution of $n \mathrm{BuLi}$ in THF ( $33 \mathrm{~mL}, 75 \mathrm{mmol}, 3$ equiv). The solution was stirred for $30 \mathrm{~min}$ at $-78{ }^{\circ} \mathrm{C}$ then transferred via cannula to a solution of 19 ( $4.7 \mathrm{~g}, 25 \mathrm{mmol}, 1$ equiv) and $\mathrm{ZnCl}_{2}$ ( $\left.4.09 \mathrm{~g}, 30 \mathrm{mmol}, 1.2 \mathrm{equiv}\right)$ in dry THF $(55 \mathrm{~mL})$. The mixture was stirred $45 \mathrm{~min}$ at $-78{ }^{\circ} \mathrm{C}$ and a solution of $18(5.82 \mathrm{~g}, 32.3 \mathrm{mmol}, 1.3 \mathrm{equiv})$ in dry THF $(35 \mathrm{~mL})$ was added via cannula. The resulting solution was stirred for $30 \mathrm{~min}$ at $-78^{\circ} \mathrm{C}$ before it was hydrolyzed with saturated $\mathrm{NH}_{4} \mathrm{Cl}$ and extracted with EtOAc. The combined organic layers were dried over $\mathrm{MgSO}_{4}$, the solvent was evaporated in vacuo, and the crude product was purified by flash chromatography $\left(\mathrm{SiO}_{2}\right.$, petroleum ether/EtOAc: 6/4). The corresponding alcohol was obtained as a colorless oil (6.94 $\left.\mathrm{g}, 75 \%\right)$. Dess-Martin periodinane $(14.1 \mathrm{~g}, 27.2 \mathrm{mmol}, 1.8 \mathrm{equiv})$ was added to a solution of this alcohol (6.70 g, 18.1 mmol, 1 equiv) in dry $\mathrm{CH}_{2} \mathrm{Cl}_{2}(160 \mathrm{~mL})$ at $0{ }^{\circ} \mathrm{C}$. After stirring $3.5 \mathrm{~h}$ at $\mathrm{rt}$, the reaction was quenched with saturated $\mathrm{NaHCO}_{3}$ and the mixture was stirred vigorously until two clear layers were obtained. The layers were separated and the aqueous layer was extracted with $\mathrm{CH}_{2} \mathrm{Cl}_{2}$. The combined organic layers were dried over $\mathrm{MgSO}_{4}$ and the solvent was removed under reduced pressure. The crude mixture was purified by flash chromatography $\left(\mathrm{SiO}_{2}\right.$, cyclohexane/EtOAc: 8/2) to give the ketone 17 as a colorless oil (1.54 g, 23\%). $\mathrm{R}_{f}$ (cyclohexane/EtOAc: 1/1, $\left.\mathrm{KMnO}_{4}, \mathrm{UV}\right)$ 0.65; ${ }^{1} \mathrm{H} \mathrm{NMR}\left(300 \mathrm{MHz}, \mathrm{CDCl}_{3}\right) \delta 7.19$ (d, J 8.7 Hz, 2H), 6.80 (d, J $8.7 \mathrm{~Hz}$, $2 \mathrm{H}), 5.65(\mathrm{~d}, J 7.4 \mathrm{~Hz}, 1 \mathrm{H}), 5.10(\mathrm{~d}, J 7.6 \mathrm{~Hz}, 1 \mathrm{H}), 4.44(\mathrm{~s}, 2 \mathrm{H}), 4.35(\mathrm{~d}, J 17.0 \mathrm{~Hz}, 1 \mathrm{H}), 4.20(\mathrm{~d}, J 17.0 \mathrm{~Hz}, 1 \mathrm{H}), 3.71$ (s, 3H), 3.65 (s, 3H), 1.35 (s, 9H); $\left.{ }^{13} \mathrm{C} \mathrm{NMR} \mathrm{(75} \mathrm{MHz,} \mathrm{CDCl} 3\right) \delta 199.7,166.6,159.4,154.7,129.6,128.7,113.7$, $80.5,72.9(2 \mathrm{C}), 60.0,55.1,53.0,28.0$.

Methyl (2R,3R)-2-[(tert-butoxycarbonyl)amino]-3-hydroxy-4-[(4-methoxybenzyl)oxy]butanoate (syn-23). (S)Synphos (2.9 mg, $4.5 \mu \mathrm{mol}, 0.011$ equiv) and [Ru(COD) $\left(\eta^{3}-2\right.$-methylallyl) 2$](1.3 \mathrm{mg}, 4.1 \mu \mathrm{mol}, 0.01$ equiv) were placed in a reaction tube and the vessel was purged with argon. Anhydrous acetone $(1.5 \mathrm{~mL}) \mathrm{previously}$ degassed by three vacuum-argon cycles was added at $r$. To this suspension was added dropwise methanolic $\mathrm{HBr}(47 \mu \mathrm{L}, 0.009$ equiv of a $0.19 \mathrm{~N}$ solution prepared by adding $48 \%$ aqueous $\mathrm{HBr}$ in degassed methanol) and the suspension was stirred at room temperature for $30 \mathrm{~min}$. The suspension immediately turned yellow, then an orange precipitate appeared and the solvent was thoroughly evaporated under vacuum to give the catalyst as an orange-brown solid $\left[\operatorname{RuBr}_{2}((S)\right.$-Synphos)], which was used directly. A solution of $\beta$-ketoester hydrochloride 17 (150 mg, $0.41 \mathrm{mmol}, 1$ equiv) in degassed anhydrous $\mathrm{CH}_{2} \mathrm{Cl}_{2}$ (1.5 mL) was then added. The reaction vessel was degassed by three vacuum-argon cycles and then placed under argon in a $250 \mathrm{~mL}$ stainless steel autoclave. The argon atmosphere was replaced with hydrogen by three cycles of pressurizing and the pressure adjusted to 120 bar. The autoclave was heated at $50{ }^{\circ} \mathrm{C}$ and stirring was maintained for $96 \mathrm{~h}$. After cooling, the reaction mixture was concentrated under reduced pressure to afford the crude $\beta$-hydroxy ester which was purified by flash chromatography $\left(\mathrm{SiO}_{2}\right.$, cyclohexane/EtOAc: 6/4) to give syn-23 (110 mg, 73\%) as a colorless oil. $\mathrm{R}_{f}$ (cyclohexane/AcOEt: $\left.1 / 1, \mathrm{KMnO}_{4}, \mathrm{UV}\right)$ 0.47; ${ }^{1} \mathrm{H} \mathrm{NMR}\left(300 \mathrm{MHz}, \mathrm{CDCl}_{3}\right) \delta$ 7.27-7.18 (m, 2H), 6.90-6.82 (m, $2 \mathrm{H}), 5.40(\mathrm{~d}, 1 \mathrm{H}, J 9.0 \mathrm{~Hz}), 4.45\left(\mathrm{~s}, 2 \mathrm{H}, \mathrm{H}_{6}\right), 4.39(\mathrm{dd}, 1 \mathrm{H}, J$ 16.1, $6.2 \mathrm{~Hz}), 4.23(\mathrm{br} \mathrm{s}, 1 \mathrm{H}), 3.78(\mathrm{~s}, 3 \mathrm{H}), 3.72(\mathrm{~s}, 3 \mathrm{H})$, 3.51 (dd, $1 \mathrm{H}, J$ 8.9, $5.4 \mathrm{~Hz}), 3.43(\mathrm{~m}, 1 \mathrm{H}), 2.98$ (br s, $1 \mathrm{H}), 1.43(\mathrm{~s}, 9 \mathrm{H}) ;{ }^{13} \mathrm{C} \mathrm{NMR}\left(75 \mathrm{MHz}, \mathrm{CDCl}_{3}\right) \delta 171.4,159.3$, 155.8, 129.6, 129.4, 113.8, 80.3, 73.1, 70.7, 70.3, 55.2, 52.5, 52.3, 28.2; HPLC : Chiralpak IA, hexane/iPrOH $95 / 5,1 \mathrm{~mL} / \mathrm{min}, \lambda=215 \mathrm{~nm} ; \mathrm{t}_{\mathrm{R}}[$ syn $]=37.71 \mathrm{~min}, \mathrm{t}_{\mathrm{R}}[\mathrm{syn}]=42.65 \mathrm{~min}, \mathrm{t}_{\mathrm{R}}[$ anti $]=50.56 \mathrm{~min}, \mathrm{t}_{\mathrm{R}}[$ anti $]=61.98$ $\min$.

Methyl (2R,3R)-2-[(tert-butoxycarbonyl)amino]-3-[(tert-butyldimethylsilyl)oxy]-4-[(4-methoxybenzyl)oxy]butanoate (syn-24). To a solution of syn-23 (97 mg, $0.26 \mathrm{mmol}, 1$ equiv) in dry DMF (0.5 mL) were added successively imidazole (54 mg, $0.79 \mathrm{mmol}, 3$ equiv) and TBSCl (99 mg, $0.66 \mathrm{mmol}, 2.5$ equiv) at rt. The mixture 
was stirred for $18 \mathrm{~h}$ at $\mathrm{rt}$, then saturated aqueous $\mathrm{NaCl}$ and $i \operatorname{Pr}_{2} \mathrm{O}$ were added. The aqueous phase was extracted with $\mathrm{Pr}_{2} \mathrm{O}$. The combined organic layers were washed with brine, dried over $\mathrm{MgSO}_{4}$, filtered and concentrated. The residue was purified by flash chromatography $\left(\mathrm{SiO}_{2}\right.$, petroleum ether/EtOAc: $\left.9 / 1\right)$ to give syn-24 (111 mg, 87\%) as a colorless oil. $\mathrm{R}_{f}$ (cyclohexane/AcOEt: 7/3, ninhydrin, UV) 0.67; ${ }^{1} \mathrm{H} \mathrm{NMR}(300 \mathrm{MHz}$, $\left.\mathrm{CDCl}_{3}\right) \delta 7.26(\mathrm{~d}, 2 \mathrm{H}, J 8.5 \mathrm{~Hz}), 6.91-6.83(\mathrm{~m}, 2 \mathrm{H}), 5.13(\mathrm{~d}, 1 \mathrm{H}, J 9.9 \mathrm{~Hz}), 4.55(\mathrm{dd}, 1 \mathrm{H}, J$ 10.0, $1.4 \mathrm{~Hz}), 4.43(\mathrm{~s}, 2 \mathrm{H})$, 4.41-4.32 (m, $1 \mathrm{H}), 3.80(\mathrm{~s}, 3 \mathrm{H}), 3.71(\mathrm{~s}, 3 \mathrm{H}), 3.43(\mathrm{dd}, 1 \mathrm{H}, J$ 9.7, $6.4 \mathrm{~Hz}), 3.35(\mathrm{dd}, 1 \mathrm{H}, J$ 9.3, $5.8 \mathrm{~Hz}), 1.47(\mathrm{~s}, 9 \mathrm{H})$, $0.82(\mathrm{~s}, 9 \mathrm{H}), 0.01(\mathrm{~s}, 3 \mathrm{H}),-0.03(\mathrm{~s}, 3 \mathrm{H}) ;{ }^{13} \mathrm{C} \mathrm{NMR}\left(75 \mathrm{MHz}, \mathrm{CDCl}_{3}\right) \delta 171.8,159.2,156.0,130.0,129.4,113.7$, $79.8,73.1,71.5,70.7,55.7,55.2,52.2,28.3,25.6,17.9,-4.5,-5.4$.

(2R,3R)-2-[(tert-Butoxycarbonyl)amino]-3-[(tert-butyldimethylsilyl)oxy]-4-[(4-methoxybenzyl)oxy]butanoic acid (syn-B'). To a solution of syn-24 (48 mg, $0.099 \mathrm{mmol}, 1$ equiv) in water/MeOH (0.2 mL:1.4 mL) was added at $\mathrm{rt}$ an aqueous solution of potassium hydroxide $(400 \mu \mathrm{L}, 0.20 \mathrm{mmol}, 2$ équiv, $0.5 \mathrm{M})$. The mixture was stirred at $40{ }^{\circ} \mathrm{C}$ for $4 \mathrm{~h}$, then quenched with a $1 \mathrm{M} \mathrm{HCl}$ solution. The aqueous layer was extracted with EtOAc. The combined organic layers were dried over $\mathrm{MgSO}_{4}$, filtered and concentrated under reduced pressure. Purification by flash chromatography $\left(\mathrm{SiO}_{2}, \mathrm{CH}_{2} \mathrm{Cl}_{2} / \mathrm{MeOH}: 8 / 2\right)$ afforded syn-B' as a colorless oil (20 mg, $\left.43 \%\right)$. $\mathrm{R}_{f}$ (cyclohexane/AcOEt: 8/2, ninhydrin, UV) 0.15; $[\alpha]_{\mathrm{D}}^{20}:-7.3\left(c 0.78, \mathrm{CHCl}_{3}\right) ;{ }^{1} \mathrm{H}$ NMR (300 MHz, MeOD) $\delta 7.30-$ $7.22(\mathrm{~m}, 2 \mathrm{H}), 6.92-6.85(\mathrm{~m}, 2 \mathrm{H}), 4.44$ (br s, 2H), 4.39 (br s, 2H), $3.78(\mathrm{~s}, 3 \mathrm{H}), 3.43-3.34(\mathrm{~m}, 2 \mathrm{H}), 1.47(\mathrm{~s}, 9 \mathrm{H}), 0.85$ (s, 9H), 0.04 (s, 3H), 0.03 (s, 3H); ${ }^{13} \mathrm{C}$ NMR (75 MHz, MeOD) $\delta$ 174.4, 160.8, 157.9, 131.3, 130.6, 114.7, 80.9, 74.0, 72.9, 71.6, 56.8, 55.6, 28.7, 26.3, 18.9, -4.3, -5.0.

Methyl (2S,3R)-2-[(tert-butoxycarbonyl)amino]-3-hydroxy-4-[(4-methoxybenzyl)oxy]butanoate (anti-23). A reaction vessel fitted with a rubber septum equipped with a balloon of argon was charged with the ester 17 (124 mg, $0.34 \mathrm{mmol}, 1$ equiv) and $\mathrm{CH}_{2} \mathrm{Cl}_{2}(2.5 \mathrm{~mL}) .126 \mu \mathrm{L}$ of a $0.0027 \mathrm{M}$ solution of Cat III $\left[\mathrm{RhCl}\left(\mathrm{Cp}^{*}\right)((R, R)-\right.$ teth-TsDPEN)] (0.1 mol\%) in $\mathrm{CH}_{2} \mathrm{Cl}_{2}$ was added and the resulting mixture was subjected to three vacuum/argon cycles before the azeotropic mixture $\mathrm{HCOOH} / \mathrm{NEt}_{3}(5 / 2)(0.17 \mathrm{~mL}, 0.68 \mathrm{mmol}, 2$ equiv) was added dropwise. The reaction mixture was stirred at $\mathrm{rt}$ for $4 \mathrm{~h}$ (monitored by TLC). Saturated $\mathrm{NaHCO}_{3}$ was added and the aqueous layer was extracted with $\mathrm{CH}_{2} \mathrm{Cl}_{2}$. The combined organic phases were dried over $\mathrm{MgSO}_{4}$, filtered and the solvent was removed under reduced pressure. The conversion and the diastereoisomeric ratio were determined by ${ }^{1} \mathrm{H}$ NMR analysis of the crude product. The crude product was purified by flash chromatography $\left(\mathrm{SiO}_{2}\right.$, pentane/EtOAc: $7 / 3$ ) to afford compound anti-23 (88 mg, 70\%). $\mathrm{R}_{f}$ (cyclohexane/AcOEt: 6/4, $\mathrm{KMnO}_{4}$, UV) 0.33; ${ }^{1} \mathrm{H}$ NMR $\left(300 \mathrm{MHz}^{\mathrm{CDCl}} \mathrm{CD}_{3} \delta 7.26-7.21(\mathrm{~m}, 2 \mathrm{H}), 6.87(\mathrm{~d}, J 8.6 \mathrm{~Hz}, 2 \mathrm{H}), 5.58(\mathrm{~d}, J 7.1 \mathrm{~Hz}\right.$, $1 \mathrm{H}), 4.45(\mathrm{~s}, 2 \mathrm{H}), 4.42-4.15(\mathrm{~m}, 2 \mathrm{H}), 3.79(\mathrm{~s}, 3 \mathrm{H}), 3.73(\mathrm{~s}, 3 \mathrm{H}), 3.55-3.41(\mathrm{~m}, 2 \mathrm{H}), 2.87(\mathrm{~d}, J 4.0 \mathrm{~Hz}, 1 \mathrm{H}), 1.43$ (s, 9H); ${ }^{13} \mathrm{C} \mathrm{NMR}\left(75 \mathrm{MHz}, \mathrm{CDCl}_{3}\right) \delta 171.4,159.3,155.9,129.6,129.5,113.8,80.0,73.1$ (2C), 70.7, 70.3, 55.2, 52.5, 28.2; HPLC : Chiralpak IA, hexane/ $\mathrm{PPOH} 95 / 5,1 \mathrm{~mL} / \mathrm{min}, \lambda=215 \mathrm{~nm} ; \mathrm{t}_{\mathrm{R}}[\mathrm{syn}]=37.71 \mathrm{~min}, \mathrm{t}_{\mathrm{R}}[$ syn] $=42.65$ $\min , \mathrm{t}_{\mathrm{R}}[\operatorname{anti-}(R, S)]=50.56 \mathrm{~min}, \mathrm{t}_{\mathrm{R}}[\operatorname{anti-}(S, R)]=61.98 \mathrm{~min}$ (major).

Methyl (2S,3R)-2-[(tert-butoxycarbonyl)amino]-3-[(tert-butyldimethylsilyl)oxy]-4-[(4-methoxybenzyl)oxy]butanoate (anti-24). To a solution of anti-23 (173 mg, $0.47 \mathrm{mmol}, 1$ equiv) in dry DMF (1 mL) were added successively imidazole ( $64 \mathrm{mg}, 0.94 \mathrm{mmol}, 2$ equiv) and TBSCl (107 mg, $0.71 \mathrm{mmol}, 1.5$ equiv) at rt. The mixture was stirred for $33 \mathrm{~h}$ at $\mathrm{rt}$, then brine and $\mathrm{Pr}_{2} \mathrm{O}$ were added. The aqueous phase was extracted with $i \mathrm{Pr}_{2} \mathrm{O}$. The combined organic layers were washed with brine, dried over $\mathrm{MgSO}_{4}$, filtered and concentrated. The residue was purified by flash chromatography $\left(\mathrm{SiO}_{2}\right.$, petroleum ether/EtOAc: 9/1) to give anti-24 (115 mg, $51 \%$ ) as a colorless oil. $\mathrm{R}_{f}$ (cyclohexane/EtOAc:8/2, ninhydrin, UV) $0.45 ;{ }^{1} \mathrm{H} \mathrm{NMR}\left(300 \mathrm{MHz}, \mathrm{CDCl}_{3}\right) \delta 7.26(\mathrm{~d}, J$ $8.2 \mathrm{~Hz}, 2 \mathrm{H}), 6.87(\mathrm{~d}, J 8.2 \mathrm{~Hz}, 1 \mathrm{H}), 5.13(\mathrm{~d}, J 10.0 \mathrm{~Hz}, 1 \mathrm{H}), 4.56-4.35(\mathrm{~m}, 2 \mathrm{H}), 4.45(\mathrm{~s}, 2 \mathrm{H}), 3.80(\mathrm{~s}, 3 \mathrm{H}), 3.72(\mathrm{~s}$, $3 \mathrm{H}), 3.46-3.33(\mathrm{~m}, 2 \mathrm{H}), 1.47(\mathrm{~s}, 9 \mathrm{H}), 0.85(\mathrm{~s}, 9 \mathrm{H}), 0.01(\mathrm{~s}, 3 \mathrm{H}),-0.03(\mathrm{~s}, 3 \mathrm{H}) ;{ }^{13} \mathrm{C} \mathrm{NMR}\left(75 \mathrm{MHz}, \mathrm{CDCl}_{3}\right) \delta 171.8$, $159.2,156.0,130.0,129.4,113.8,79.8,73.1,71.6,70.7,55.7,55.2,52.2,29.7,28.3,25.7,-4.5,-5.4$. 
(2S,3R)-2-[(tert-Butoxycarbonyl)amino]-3-[(tert-butyldimethylsilyl)oxy]-4-[(4-methoxybenzyl)oxy]butanoic acid (anti-B'). To a solution of anti-24 (93 mg, $0.19 \mathrm{mmol}, 1$ equiv) in THF ( $3 \mathrm{~mL}$ ) was added at rt a $2 \mathrm{M}$ solution of TMSOK (0.76 mL, $1.52 \mathrm{mmol}, 8$ equiv) in THF. After $1.5 \mathrm{~h}$, the reaction medium was quenched with a $10 \%$ aqueous solution of citric acid $(5 \mathrm{~mL})$. The phases were separated and the aqueous layer was extracted with EtOAc. The combined organic layers were washed with brine, dried over $\mathrm{MgSO}_{4}$ and concentrated under reduced pressure. Purification by flash chromatography $\left(\mathrm{SiO}_{2}, \mathrm{CH}_{2} \mathrm{Cl}_{2} / \mathrm{MeOH}\right.$ : 9/1) afforded anti-B' as a pale yellow oil (59 mg, 66\%). $\mathrm{R}_{f}\left(\mathrm{CH}_{2} \mathrm{Cl}_{2} / \mathrm{MeOH}: 9 / 1\right.$, ninhydrin, UV) 0.26; ${ }^{1} \mathrm{H} \mathrm{NMR}(300 \mathrm{MHz}, \mathrm{MeOD}) \delta 7.30-7.22$ $(\mathrm{m}, 2 \mathrm{H}), 6.92-6.85(\mathrm{~m}, 2 \mathrm{H}), 4.44$ (bs, 2H), 4.39 (bs, 2H), $3.78(\mathrm{~s}, 3 \mathrm{H}), 3.43-3.34(\mathrm{~m}, 2 \mathrm{H}), 1.47(\mathrm{~s}, 9 \mathrm{H}), 0.85(\mathrm{~s}$, 9H), 0.04 (s, 3H), 0.03 (s, 3H); ${ }^{13} \mathrm{C} \mathrm{NMR} \mathrm{(75} \mathrm{MHz,} \mathrm{MeOD)} \delta 174.4,160.8,157.9,131.3,130.6,114.7,80.9,74.0$, $72.9,71.6,56.8,55.6,28.7,26.3,18.9,-4.3,-5.0$.

Methyl (R)-3-(benzyloxy)-2-methylpropanoate (26). To a solution of $(R)$-Roche ester $\mathbf{2 5}$ (200 mg, $1.69 \mathrm{mmol}, 1$ equiv) in $\mathrm{CH}_{2} \mathrm{Cl}_{2}(3 \mathrm{~mL})$ was added at $0{ }^{\circ} \mathrm{C}$ benzyl trichloroacetimidate $(640 \mathrm{mg}, 2.54 \mathrm{mmol}, 1.5 \mathrm{equiv})$. A solution of $\mathrm{CF}_{3} \mathrm{SO}_{3} \mathrm{H}$ in $\mathrm{CH}_{2} \mathrm{Cl}_{2}\left(0.85 \mathrm{M}, 100 \mu \mathrm{L}, 0.09 \mathrm{mmol}, 0.05\right.$ equiv) was added at $0{ }^{\circ} \mathrm{C}$ and the mixture was stirred at room temperature for $44 \mathrm{~h}$. After filtration, the filtrate was concentrated under reduced pressure and saturated aqueous $\mathrm{NaHCO}_{3}(15 \mathrm{~mL})$ was added. The aqueous layer was extracted with EtOAc, the combined organic layers were washed with brine, dried over $\mathrm{MgSO}_{4}$ and concentrated under reduced pressure. The crude mixture was purified by flash column chromatography $\left(\mathrm{SiO}_{2}\right.$, petroleum ether/Et $\left.\mathrm{t}_{2} \mathrm{O}: 95 / 5\right)$ to afford the desired product 26 (262 mg, 74\%) as a yellow oil. $\mathrm{R}_{f}$ (cyclohexane/EtOAc: 9/1, UV, $\mathrm{KMnO}_{4}$ ) 0.30; $[\alpha]_{\mathrm{D}}^{20}-8.0$ (c 1.60, $\left.\mathrm{CHCl}_{3}\right) ;{ }^{1} \mathrm{H} \mathrm{NMR}\left(\mathrm{CDCl}_{3}, 300 \mathrm{MHz}\right) \delta=7.38-7.24(\mathrm{~m}, 5 \mathrm{H}), 4.53(\mathrm{~s}, 2 \mathrm{H}), 3.70(\mathrm{~s}, 3 \mathrm{H}), 3.66(\mathrm{dd}$, J 9.2, 7.3 Hz, 1H), 3.50 (dd, J 9.1, $5.9 \mathrm{~Hz}, 1 \mathrm{H}), 2.86-2.72(\mathrm{~m}, 1 \mathrm{H}), 1.18(\mathrm{~d}, J 7.1 \mathrm{~Hz}, 3 \mathrm{H}) ;{ }^{13} \mathrm{C} \mathrm{NMR}\left(\mathrm{CDCl}_{3}, 75\right.$ $\mathrm{MHz}) \delta 175.4,138.3,128.5,127.7$ (2C), 73.2, 72.1, 51.8, 40.3, 14.1.

(S)-3-(Benzyloxy)-2-methylpropan-1-ol (27). To a suspension of $\mathrm{LiAlH}_{4}$ (287 mg, $7.56 \mathrm{mmol}, 1.5$ equiv) in $\mathrm{Et}_{2} \mathrm{O}$ $(7.5 \mathrm{~mL})$ was added dropwise a solution of $26\left(1.05 \mathrm{~g}, 5.04 \mathrm{mmol}, 1\right.$ equiv) in $\mathrm{Et}_{2} \mathrm{O}(7.5 \mathrm{~mL})$ at $0{ }^{\circ} \mathrm{C}$. The mixture was stirred for $1.5 \mathrm{~h}$ and $600 \mathrm{mg}$ of $\mathrm{SiO}_{2}$ were added at $0{ }^{\circ} \mathrm{C}$, followed by water $(1 \mathrm{~mL})$, an aqueous solution of $15 \% \mathrm{NaOH}(3 \mathrm{~mL})$ and water again $(1 \mathrm{~mL}) . \mathrm{MgSO}_{4}$ was added, the mixture was filtered and the solvent was removed under reduced pressure to afford the desired product 27 (866 mg, 95\%) as a colorless oil. $\mathrm{R}_{f}$ (cyclohexane/EtOAc: 8/2, $\mathrm{KMnO}_{4}$ ) 0.19; $[\alpha]_{\mathrm{D}}^{20}-22.2$ (c 0.45, $\mathrm{CHCl}_{3}$ ); ${ }^{1} \mathrm{H} \mathrm{NMR}\left(\mathrm{CDCl}_{3}, 300 \mathrm{MHz}\right) \delta 7.39-7.24$ (m, $5 \mathrm{H}), 4.52(\mathrm{~s}, 2 \mathrm{H}), 3.68-3.52(\mathrm{~m}, 3 \mathrm{H}), 3.43(\mathrm{dd}, J 9.0,8.1 \mathrm{~Hz}, 1 \mathrm{H}), 2.52(\mathrm{~s}, 1 \mathrm{H}), 2.15-2.02(\mathrm{~m}, 1 \mathrm{H}), 0.89(\mathrm{~d}, J 7.0$ $\mathrm{Hz}, 3 \mathrm{H}) ;{ }^{13} \mathrm{C} \mathrm{NMR}\left(\mathrm{CDCl}_{3}, 75 \mathrm{MHz}\right) \delta 138.2,128.6,127.9,127.7,75.6,73.5,68.0,35.7,13.6$.

$(\boldsymbol{R})$-[(3-lodo-2-methylpropoxy)methyl]benzene (28). To a solution of $\mathrm{PPh}_{3}(1.40 \mathrm{~g}, 5.33 \mathrm{mmol}, 1.2$ equiv) in $\mathrm{CH}_{2} \mathrm{Cl}_{2}\left(15 \mathrm{~mL}\right.$ ) cooled to $0{ }^{\circ} \mathrm{C}$ were successively added imidazole (453 mg, $6.66 \mathrm{mmol}, 1.5 \mathrm{equiv}$ ) and $\mathrm{I}_{2}$ (1.52 g, $5.99 \mathrm{mmol}, 1.35$ equiv). A solution of 27 ( $800 \mathrm{mg}, 4.44 \mathrm{mmol}, 1$ equiv) in $\mathrm{CH}_{2} \mathrm{Cl}_{2}(2.5 \mathrm{~mL})$ was added and the mixture was stirred at room temperature for $2 \mathrm{~h}$. The solvent was removed under reduced pressure and saturated aqueous $\mathrm{Na}_{2} \mathrm{~S}_{2} \mathrm{O}_{3}$ was added. The aqueous layer was extracted with $\mathrm{Et}_{2} \mathrm{O}$ and the combined organic layers were washed with water and brine, dried over $\mathrm{MgSO}_{4}$ and concentrated under reduced pressure. Pentane was added, the suspension was filtered and the solvent was removed under reduced pressure. The crude mixture was purified by flash column chromatography $\left(\mathrm{SiO}_{2}\right.$, petroleum ether/toluene: $\left.83 / 7\right)$ to afford the desired product 28 (1.04 g, 81\%) as a yellow oil. $\mathrm{R}_{f}$ (cyclohexane/EtOAc: 98/2, phosphomolybdic acid) 0.23; ${ }^{1} \mathrm{H} \mathrm{NMR}\left(\mathrm{CDCl}_{3}, 300 \mathrm{MHz}\right) \delta 7.40-7.24(\mathrm{~m}, 5 \mathrm{H}), 4.53(\mathrm{~s}, 2 \mathrm{H}), 3.40$ (dd, J 9.3, $\left.5.2 \mathrm{~Hz}, 1 \mathrm{H}\right), 3.35-3.26(\mathrm{~m}, 3 \mathrm{H})$, $1.87-1.70(\mathrm{~m}, 1 \mathrm{H}), 1.00(\mathrm{~d}, J 6.7 \mathrm{~Hz}, 3 \mathrm{H}) ;{ }^{13} \mathrm{C} \mathrm{NMR}\left(\mathrm{CDCl}_{3}, 75 \mathrm{MHz}\right) \delta$ 138.5, 128.5, $127.8(2 \mathrm{C}), 74.3,73.3,35.3$, $17.8,14.1$.

Methyl (S)-7-(benzyloxy)-6-methyl-3-oxoheptanoate (29). To a suspension of $\mathrm{NaH}$ (60\% dispersion in mineral oil) (91 mg, $2.27 \mathrm{mmol}, 1.5$ equiv) in THF (3 mL) was added dropwise methyl acetoacetate $(0.16 \mathrm{~mL}, 1.52$ mmol, 1 equiv) at $0^{\circ} \mathrm{C}$. The mixture was stirred at $0{ }^{\circ} \mathrm{C}$ for $10 \mathrm{~min}$ and a $2.28 \mathrm{M}$ solution of $n \mathrm{BuLi}(0.73 \mathrm{~mL}, 1.67$ 
mmol, 1.1 equiv) was added dropwise. The mixture was stirred at $0{ }^{\circ} \mathrm{C}$ for $10 \mathrm{~min}$ and a solution of 28 (484 mg, $1.67 \mathrm{mmol}, 1.1$ equiv) in THF $(2.5 \mathrm{~mL})$ was added dropwise. The mixture was stirred at room temperature for $23 \mathrm{~h}$. A $10 \%$ aqueous solution of $\mathrm{HCl}(8 \mathrm{~mL})$ and AcOEt $(8 \mathrm{~mL})$ were added and the aqueous layer was extracted with EtOAc. The combined organic layers were washed with water and brine, dried over $\mathrm{MgSO}_{4}$ and concentrated under reduced pressure. The crude mixture was purified by flash column chromatography $\left(\mathrm{SiO}_{2}\right.$, petroleum ether/EtOAc: 98/2 then 85/15) to afford the desired product $29(238 \mathrm{mg}, 56 \%)$ as a yellow oil. $\mathrm{R}_{f}$ (cyclohexane/EtOAc: 8/2, $\mathrm{KMnO}_{4}$ ) 0.40; $[\alpha]_{\mathrm{D}}^{20}-5.8\left(\mathrm{c} 0.60, \mathrm{CHCl}_{3}\right) ;{ }^{1} \mathrm{H} \mathrm{NMR}\left(\mathrm{CDCl}_{3}, 300 \mathrm{MHz}\right) \delta 7.38-7.25(\mathrm{~m}$, $5 \mathrm{H}), 4.48(\mathrm{~s}, 2 \mathrm{H}), 3.73(\mathrm{~s}, 3 \mathrm{H}), 3.43(\mathrm{~s}, 2 \mathrm{H}), 3.29(\mathrm{~d}, J 5.9 \mathrm{~Hz}, 2 \mathrm{H}), 2.68-2.47(\mathrm{~m}, 2 \mathrm{H}), 1.85-1.68(\mathrm{~m}, 2 \mathrm{H}), 1.57-$ $1.40(\mathrm{~m}, 1 \mathrm{H}), 0.92(\mathrm{~d}, J 6.7 \mathrm{~Hz}, 3 \mathrm{H}) ;{ }^{13} \mathrm{C} \mathrm{NMR}\left(\mathrm{CDCl}_{3}, 75 \mathrm{MHz}\right) \delta 202.9,167.8,138.7,128.5,127.7,127.7,75.6$, $73.2,52.5,49.1,40.9,33.0,27.5,17.1$.

Methyl (3R,6S)-7-(benzyloxy)-3-hydroxy-6-methylheptanoate (R,S)-(30). Ru(COD) $\left[\eta^{3}-\left(\mathrm{CH}_{2}\right)_{2} \mathrm{CHCH}_{3}\right]_{2}(4.1 \mathrm{mg}$, $0.0129 \mathrm{mmol}, 0.02$ equiv.) and ( $R$ )-SYNPHOS (9.1 $\mathrm{mg}, 0.0142 \mathrm{mmol}, 0.022$ equiv.) were placed in a Schlenk tube, which was purged with argon. Anhydrous acetone $(0.7 \mathrm{~mL})$, previously degassed by three vacuum-argon cycles, and a $0.165 \mathrm{M}$ solution of methanolic hydrobromic acid $(0.17 \mathrm{~mL}, 0.0285 \mathrm{mmol}, 0.044$ equiv.) were successively added. The suspension was stirred at room temperature for $30 \mathrm{~min}$. The solvent was evaporated under vacuum to provide the catalyst as an orange solid. A solution of 29 (180 $\mathrm{mg}, 0.647 \mathrm{mmol}, 1$ equiv) in previously degassed $\mathrm{MeOH}(1 \mathrm{~mL})$ was added and the mixture was stirred under 11 bar of $\mathrm{H}_{2}$ at $50{ }^{\circ} \mathrm{C}$ for $22 \mathrm{~h}$. The solvent was evaporated under reduced pressure and the crude mixture was purified by flash column chromatography (petroleum ether/AcOEt: 8/2) to afford the desired product $(R, S)-30(168 \mathrm{mg}, 93 \%)$ as a yellow oil. $\mathrm{R}_{f}$ (cyclohexane/EtOAc: 7/3, $\mathrm{KMnO}_{4}$ ) 0.28; $[\alpha]_{\mathrm{D}}^{20}-14.8$ (c $0.50, \mathrm{CHCl}_{3}$ ); ${ }^{1} \mathrm{H} \mathrm{NMR}\left(\mathrm{C}_{6} \mathrm{D}_{6}, 300 \mathrm{MHz}\right) \delta$ $7.34-7.28(\mathrm{~m}, 2 \mathrm{H}), 7.23-7.06(\mathrm{~m}, 3 \mathrm{H}), 4.33(\mathrm{~s}, 2 \mathrm{H}), 3.91-3.80(\mathrm{~m}, 1 \mathrm{H}), 3.28(\mathrm{~s}, 3 \mathrm{H}), 3.17(\mathrm{dd}, J 8.9,5.8 \mathrm{~Hz}$, $1 \mathrm{H}), 3.10(\mathrm{dd}, J 8.9,6.2 \mathrm{~Hz}, 1 \mathrm{H}), 2.78(\mathrm{~d}, J 3.7 \mathrm{~Hz}, 1 \mathrm{H}), 2.22(\mathrm{dd}, J 16.2,8.0 \mathrm{~Hz}, 1 \mathrm{H}), 2.15(\mathrm{dd}, J 16.2,4.2 \mathrm{~Hz}, 1 \mathrm{H})$, $1.79-1.54(\mathrm{~m}, 2 \mathrm{H}), 1.43-1.22(\mathrm{~m}, 2 \mathrm{H}), 1.16-1.01(\mathrm{~m}, 1 \mathrm{H}), 0.91(\mathrm{~d}, J 6.6 \mathrm{~Hz}, 3 \mathrm{H}) ;{ }^{13} \mathrm{C} \mathrm{NMR}\left(\mathrm{C}_{6} \mathrm{D}_{6}, 75 \mathrm{MHz}\right) \delta$ $173.2,139.5,128.6,127.8,127.6,75.8,73.2,68.4,51.1,41.5,34.4,33.9,29.9,17.5$.

Methyl (3S,6S)-7-(benzyloxy)-3-hydroxy-6-methylheptanoate $(S, S)$-(30). (S,S)-30 was obtained in $87 \%$ yield using $(S)$-SYNPHOS and the same protocol as for $(R, S)-30 . \mathrm{R}_{f}$ (cyclohexane/EtOAc: $\left.7 / 3, \mathrm{KMnO}_{4}\right) 0.33 ;[\alpha]_{\mathrm{D}}^{20}+6.8$ (c 0.40, $\left.\mathrm{CHCl}_{3}\right) ;{ }^{1} \mathrm{H} \mathrm{NMR}\left(\mathrm{C}_{6} \mathrm{D}_{6}, 300 \mathrm{MHz}\right) \delta 7.34-7.28(\mathrm{~m}, 2 \mathrm{H}), 7.23-7.06(\mathrm{~m}, 3 \mathrm{H}), 4.33(\mathrm{~s}, 2 \mathrm{H}), 3.92-3.79(\mathrm{~m}$, $1 \mathrm{H}), 3.27(\mathrm{~s}, 3 \mathrm{H}), 3.17(\mathrm{dd}, J 8.9,6.0 \mathrm{~Hz}, 1 \mathrm{H}), 3.11(\mathrm{dd}, J 8.9,6.3 \mathrm{~Hz}, 1 \mathrm{H}), 2.75(\mathrm{~s}, 1 \mathrm{H}), 2.22(\mathrm{dd}, J 16.2,8.3 \mathrm{~Hz}$, $1 \mathrm{H}), 2.14(\mathrm{dd}, J 16.2,4.0 \mathrm{~Hz}, 1 \mathrm{H}), 1.78-1.61(\mathrm{~m}, 1 \mathrm{H}), 1.52-1.08(\mathrm{~m}, 4 \mathrm{H}), 0.90(\mathrm{~d}, J 6.7 \mathrm{~Hz}, 3 \mathrm{H}) ;{ }^{13} \mathrm{C} \mathrm{NMR}\left(\mathrm{C}_{6} \mathrm{D}_{6}\right.$, $75 \mathrm{MHz}) \delta 173.1,139.5,128.4,127.8,127.7,75.9,73.2,68.3,51.1,41.6,33.8,33.8,29.8,17.4$.

Methyl (3R,6S)-7-(benzyloxy)-3-[(tert-butyldimethylsilyl)oxy]-6-methylheptanoate $(R, S)$-(31). To a solution of $(R, S)$-30 (137 mg, 0.49 mmol, 1 equiv) in $\mathrm{CH}_{2} \mathrm{Cl}_{2}(3 \mathrm{~mL})$ cooled to $0{ }^{\circ} \mathrm{C}$ were successively added 2,6-lutidine ( $0.45 \mathrm{~mL}, 1.96 \mathrm{mmol}, 4$ equiv) and TBSOTf $\left(0.40 \mathrm{~mL}, 3.42 \mathrm{mmol}, 7\right.$ equiv). The mixture was stirred at $0{ }^{\circ} \mathrm{C}$ for 3 $\mathrm{h}$ and quenched by saturated aqueous $\mathrm{NH}_{4} \mathrm{Cl}$ at $0{ }^{\circ} \mathrm{C}$. The aqueous layer was extracted with $\mathrm{CH}_{2} \mathrm{Cl}_{2}, \mathrm{the}$ combined organic layers were washed with brine, dried over $\mathrm{MgSO}_{4}$ and concentrated under reduced pressure. The crude mixture was purified by silica gel column chromatography (pentane/AcOEt: 97/3) to afford the desired product $(R, S)-31(159 \mathrm{mg}, 82 \%)$ as a yellow oil. $\mathrm{R}_{f}$ (cyclohexane/AcOEt: $\left.7 / 3, \mathrm{KMnO}_{4}\right)$ 0.75; $[\alpha]_{\mathrm{D}}^{20}:-15.8\left(\mathrm{c} 0.45, \mathrm{CHCl}_{3}\right) .{ }^{1} \mathrm{H}$ NMR $\left(\mathrm{C}_{6} \mathrm{D}_{6}, 300 \mathrm{MHz}\right) \delta 7.34-7.28(\mathrm{~m}, 2 \mathrm{H}), 7.24-7.06(\mathrm{~m}, 3 \mathrm{H}), 4.33(\mathrm{~s}, 2 \mathrm{H})$, $4.26-4.16(\mathrm{~m}, 1 \mathrm{H}), 3.37(\mathrm{~s}, 3 \mathrm{H}), 3.17(\mathrm{dd}, J 8.9,6.0 \mathrm{~Hz}, 1 \mathrm{H}), 3.12(\mathrm{dd}, J 8.9,6.2 \mathrm{~Hz}, 1 \mathrm{H}), 2.45(\mathrm{dd}, J 14.7,7.7 \mathrm{~Hz}$, $1 \mathrm{H}), 2.29(\mathrm{dd}, J 14.7,4.8 \mathrm{~Hz}, 1 \mathrm{H}), 1.78-1.62(\mathrm{~m}, 1 \mathrm{H}), 1.62-1.40(\mathrm{~m}, 3 \mathrm{H}), 1.22-1.04(\mathrm{~m}, 1 \mathrm{H}), 0.98(\mathrm{~s}, 9 \mathrm{H}), 0.92(\mathrm{~d}$, J $6.7 \mathrm{~Hz}, 3 \mathrm{H}), 0.11(\mathrm{~s}, 3 \mathrm{H}), 0.10(\mathrm{~s}, 3 \mathrm{H}) ;{ }^{13} \mathrm{C} N M R\left(\mathrm{C}_{6} \mathrm{D}_{6}, 75 \mathrm{MHz}\right) \delta 171.7,139.5,128.6,128.4,127.7,75.8,73.2$, 70.1, 51.0, 42.5, 35.3, 34.0, 29.2, 26.1, 18.3, 17.4, -4.3, -4.6.

Methyl (3S,6S)-7-(benzyloxy)-3-((tert-butyldimethylsilyl)oxy)-6-methylheptanoate (S,S)-(31). Compound $(S, S)$-31 was obtained in $96 \%$ yield following the same protocol as for compound $(S, R)-31$. $R_{f}$ 
(cyclohexane/AcOEt: 7/3, $\mathrm{KMnO}_{4}$ ) 0.68; $[\alpha]_{\mathrm{D}}^{20}:+11.5$ (c $\left.0.75, \mathrm{CHCl}_{3}\right) ;{ }^{1} \mathrm{H} \mathrm{NMR}\left(\mathrm{C}_{6} \mathrm{D}_{6}, 300 \mathrm{MHz}\right) \delta 7.34-7.28(\mathrm{~m}$, $2 \mathrm{H}), 7.24-7.06(\mathrm{~m}, 3 \mathrm{H}), 4.33(\mathrm{~s}, 2 \mathrm{H}), 4.25-4.15(\mathrm{~m}, 1 \mathrm{H}), 3.37(\mathrm{~s}, 3 \mathrm{H}), 3.18(\mathrm{dd}, J 8.9,6.0 \mathrm{~Hz}, 1 \mathrm{H}), 3.12(\mathrm{dd}, J$ 8.9, 6.2 Hz, 1H), 2.45 (dd, J 14.7, 7.7 Hz, 1H), 2.29 (dd, J 14.7, $4.8 \mathrm{~Hz}, 1 \mathrm{H}), 1.78-1.62\left(\mathrm{~m}, 1 \mathrm{H}, \mathrm{H}_{7}\right), 1.59-1.40$ $(\mathrm{m}, 3 \mathrm{H}), 1.22-1.04(\mathrm{~m}, 1 \mathrm{H}), 0.98(\mathrm{~s}, 9 \mathrm{H}), 0.92(\mathrm{~d}, J 6.7 \mathrm{~Hz}, 3 \mathrm{H}), 0.11(\mathrm{~s}, 3 \mathrm{H}), 0.11(\mathrm{~s}, 3 \mathrm{H}) ;{ }^{13} \mathrm{C} \mathrm{NMR}\left(\mathrm{C}_{6} \mathrm{D}_{6}, 75\right.$ $\mathrm{MHz}) \delta 171.7,139.5,128.6,127.7,127.6,75.8,73.2,70.1,51.0,42.6,35.3,34.0,29.2,26.1,18.3,17.3,-4.3,-$ 4.6.

Methyl (3R,6S)-3,7-dihydroxy-6-methylheptanoate $(R, S)$-(32). To a solution of $(R, S)$-31 (138 $\mathrm{mg}, 0.35 \mathrm{mmol}, 1$ equiv) in THF ( $1.8 \mathrm{~mL}$ ) was added palladium on carbon $10 \%$ (56 mg, $0.05 \mathrm{mmol}, 0.15$ equiv). The medium was purged with argon and three vaccum- $\mathrm{H}_{2}$ cycles were made. The mixture was stirred at room temperature for $22 \mathrm{~h}$ under $1 \mathrm{~atm}$ of $\mathrm{H}_{2}$, filtered on celite and concentrated under reduced pressure. The crude mixture was purified on silica gel column chromatography (petroleum ether/MTBE: 6/4) to afford the desired product $(R, S)$ $32(102 \mathrm{mg}, 95 \%)$ as a colorless oil. $\mathrm{R}_{f}$ (cyclohexane/AcOEt: $\left.7 / 3, \mathrm{KMnO}_{4}\right) 0.38 ;[\alpha]_{\mathrm{D}}^{20}$ : $-17.8\left(\mathrm{c} 0.75, \mathrm{CHCl}_{3}\right) ;{ }^{1} \mathrm{H}$ $\operatorname{NMR}\left(\mathrm{C}_{6} \mathrm{D}_{6}, 300 \mathrm{MHz}\right) \delta 4.24-4.14(\mathrm{~m}, 1 \mathrm{H}), 3.38(\mathrm{~s}, 3 \mathrm{H}), 3.21$ (dd, J 10.4, $\left.5.7 \mathrm{~Hz}, 1 \mathrm{H}\right), 3.15$ (dd, J 10.4, 5.9 Hz, $1 \mathrm{H}), 2.44(\mathrm{dd}, J 14.7,7.6 \mathrm{~Hz}, 1 \mathrm{H}), 2.29(\mathrm{dd}, J 14.7,4.8 \mathrm{~Hz}, 1 \mathrm{H}), 1.59-1.26(\mathrm{~m}, 4 \mathrm{H}), 1.12-1.00(\mathrm{~m}, 1 \mathrm{H}), 0.98(\mathrm{~s}$, $9 \mathrm{H}), 0.82(\mathrm{~d}, J 6.6 \mathrm{~Hz}, 3 \mathrm{H}), 0.11(\mathrm{~s}, 3 \mathrm{H}), 0.10(\mathrm{~s}, 3 \mathrm{H}) ;{ }^{13} \mathrm{C} N M R\left(\mathrm{C}_{6} \mathrm{D}_{6}, 75 \mathrm{MHz}\right) \delta 171.8,70.0,67.8,51.1,42.4$, 36.0, 35.2, 28.6, 26.1, 18.3, 16.8, $-4.3,-4.6$.

Methyl (3S,6S)-3,7-dihydroxy-6-methylheptanoate $(S, S)-(32)$. Compound $(S, S)$-32 was obtained in 93\% yield following the same procedure than for compound $(S, R)-32$. $\mathrm{R}_{f}$ (cyclohexane/AcOEt: 7/3, $\left.\mathrm{KMnO}_{4}\right) 0.43 ;[\alpha]_{\mathrm{D}}^{20}$ : +13.7 (c $\left.0.80, \mathrm{CHCl}_{3}\right) ;{ }^{1} \mathrm{H}$ NMR $\left(\mathrm{C}_{6} \mathrm{D}_{6}, 300 \mathrm{MHz}\right) \delta 4.23-4.13(\mathrm{~m}, 1 \mathrm{H}), 3.37(\mathrm{~s}, 3 \mathrm{H}), 3.23(\mathrm{dd}, J 10.3,5.5 \mathrm{~Hz}, 1 \mathrm{H})$, $3.16(\mathrm{dd}, J$ 10.3, $6.0 \mathrm{~Hz}, 1 \mathrm{H}), 2.45$ (dd, J 14.7, 7.8 Hz, 1H), 2.28 (dd, J 14.7, $5.0 \mathrm{~Hz}, 1 \mathrm{H}), 1.58-1.24(\mathrm{~m}, 4 \mathrm{H}), 1.13$ $-1.00(\mathrm{~m}, 1 \mathrm{H}), 0.98(\mathrm{~s}, 9 \mathrm{H}), 0.82(\mathrm{~d}, J 6.6 \mathrm{~Hz}, 3 \mathrm{H}), 0.11(\mathrm{~s}, 3 \mathrm{H}), 0.10(\mathrm{~s}, 3 \mathrm{H}) ;{ }^{13} \mathrm{C} N M R\left(\mathrm{C}_{6} \mathrm{D}_{6}, 75 \mathrm{MHz}\right) \delta 171.8$, 70.1, 67.8, 51.1, 42.5, 36.1, 35.2, 28.6, 26.1, 18.3, 16.8, -4.4, -4.6.

Methyl $(3 R, 6 S)$-3-hydroxy-6-methyl-7-oxoheptanoate $(R, S)-(33)$. To a solution of $(R, S)$-32 (82 mg, $0.27 \mathrm{mmol}$, 1 equiv) in $\mathrm{CH}_{2} \mathrm{Cl}_{2}(1 \mathrm{~mL})$ was added DMP (171 mg, $0.40 \mathrm{mmol}, 1.5$ equiv) and the mixture was stirred at room temperature for $3 \mathrm{~h}$. After dilution with $\mathrm{Et}_{2} \mathrm{O}$, the organic layer was successively washed with saturated aqueous $\mathrm{Na}_{2} \mathrm{~S}_{2} \mathrm{O}_{3}$ and a saturated solution of $\mathrm{NaHCO}_{3}$. The aqueous layer was extracted with AcOEt, the combined organic layers were washed with brine, dried over $\mathrm{MgSO}_{4}$ and concentrated under reduced pressure. The crude mixture was purified by silica gel column chromatography (petroleum ether/i $\operatorname{Pr}_{2} \mathrm{O}: 8 / 2$ ) to afford the desired product $(R, S)-33$ (52 mg, 64\%) as a colorless oil. $\mathrm{R}_{f}$ (cyclohexane/AcOEt: 6/4, $\mathrm{KMnO}_{4}$ ) 0.68; ${ }^{1} \mathrm{H}$ NMR $\left(\mathrm{C}_{6} \mathrm{D}_{6}, 300 \mathrm{MHz}\right) \delta 9.26(\mathrm{~d}, J 1.6 \mathrm{~Hz}, 1 \mathrm{H}), 4.17-4.06(\mathrm{~m}, 1 \mathrm{H}), 3.37(\mathrm{~s}, 3 \mathrm{H}), 2.38(\mathrm{dd}, J 14.8,7.4 \mathrm{~Hz}, 1 \mathrm{H})$, 2.19 (dd, J 14.8, 5.0 Hz, 1H), 1.78 (sxtd, J 6.9, $1.5 \mathrm{~Hz}, 1 \mathrm{H}), 1.56-1.44(\mathrm{~m}, 1 \mathrm{H}), 1.39-1.25(\mathrm{~m}, 2 \mathrm{H}), 1.19-1.05(\mathrm{~m}$, $1 \mathrm{H}), 0.95(\mathrm{~s}, 9 \mathrm{H}), 0.75(\mathrm{~d}, J 7.1 \mathrm{~Hz}, 3 \mathrm{H}), 0.09(\mathrm{~s}, 3 \mathrm{H}), 0.06(\mathrm{~s}, 3 \mathrm{H}) ;{ }^{13} \mathrm{C} N M R\left(\mathrm{C}_{6} \mathrm{D}_{6}, 75 \mathrm{MHz}\right) \delta 203.0,171.5,69.5$, $51.1,46.1,42.3,34.9,26.0,25.8,18.2,13.3,-4.4,-4.6$.

Methyl (3S,6S)-3-hydroxy-6-methyl-7-oxoheptanoate $(S, S)-(33)$. Compound $(S, S)-33$ was obtained in $67 \%$ yield following the same procedure than for compound $(S, R)-33$. $\mathrm{R}_{f}$ (cyclohexane/AcOEt: 6/4, $\mathrm{KMnO}_{4}$ ) 0.62; $[\alpha]_{\mathrm{D}}^{20}$ : +12.0 (c 0.40, $\left.\mathrm{CHCl}_{3}\right) ;{ }^{1} \mathrm{H} \mathrm{NMR}\left(\mathrm{C}_{6} \mathrm{D}_{6}, 300 \mathrm{MHz}\right) \delta 9.27(\mathrm{~d}, J 1.6 \mathrm{~Hz}, 1 \mathrm{H}), 4.16-4.06(\mathrm{~m}, 1 \mathrm{H}), 3.37(\mathrm{~s}, 3 \mathrm{H})$, 2.39 (dd, J 14.8, 7.4 Hz, 1H), 2.19 (dd, J 14.8, 5.1 Hz, 1H), 1.79 (sxtd, J 6.5, $1.4 \mathrm{~Hz}, 1 \mathrm{H}), 1.56-1.41(\mathrm{~m}, 1 \mathrm{H}), 1.40$ $-1.24(\mathrm{~m}, 2 \mathrm{H}), 1.20-1.06(\mathrm{~m}, 1 \mathrm{H}), 0.95(\mathrm{~s}, 9 \mathrm{H}), 0.74(\mathrm{~d}, J 7.0 \mathrm{~Hz}, 3 \mathrm{H}), 0.09(\mathrm{~s}, 3 \mathrm{H}), 0.06(\mathrm{~s}, 3 \mathrm{H}) ;{ }^{13} \mathrm{C} \mathrm{NMR}\left(\mathrm{C}_{6} \mathrm{D}_{6}\right.$, $75 \mathrm{MHz}) \delta$ 203.0, 171.5, 69.5, 51.1, 46.1, 42.4, 34.8, 26.0, 25.9, 18.2, 13.2, -4.4, -4.6.

Methyl $\quad(3 S, 6 S, 10 R, 11 R, 12 R, 13 R)(E)-3,11,13-t r i s[(t e r t-b u t y l d i m e t h y l s i l y l) o x y]-12-\{(6 S, 7 S)(E)-7-[(t e r t-b u t y l-$ dimethylsilyl)oxy]-4,4,6,8-tetramethyl-5-oxonon-2-enamido\}-7-hydroxy-6,8,10,14-tetramethylpentadec-8-

enoate (35). To a solution of 34 (145 mg, $0.16 \mathrm{mmol}, 1.4$ equiv) and (S,S)-33 (36 mg, $0.12 \mathrm{mmol}, 1$ equiv) in freshly distilled DMSO $(4.1 \mathrm{~mL})$ were successively added $\mathrm{CrCl}_{2}\left(230 \mathrm{mg}, 1.87 \mathrm{mmol}, 16\right.$ equiv) and $\mathrm{NiCl}_{2}(\mathrm{dppe})$ ( $3.8 \mathrm{mg}, 0.007 \mathrm{mmol}, 0.06$ equiv). Three vacuum/argon cycles were performed and the mixture was stirred at 
room temperature for $21 \mathrm{~h}$. A mixture of sodium serinate/AcOEt $1 / 1(20 \mathrm{~mL})$ was added at $0{ }^{\circ} \mathrm{C}$ and the medium was stirred at room temperature for $1 \mathrm{~h}$. After filtration, the aqueous layer was extracted with AcOEt, the combined organic layers were washed with water and brine, dried over $\mathrm{MgSO}_{4}$ and concentrated under reduced pressure. The crude mixture was purified by silica gel column chromatography (pentane/i $\operatorname{Pr}_{2} \mathrm{O}: 8 / 2$ to $7 / 3$ and $5 / 5$ ) to afford the minor diastereomer $(8 \mathrm{mg}$ ) and the major diastereomer $(24 \mathrm{mg}$ ) as yellow oils (total yield: $25 \%)$.

Major diastereomer: $\mathrm{R}_{f}$ (cyclohexane/iPr $2 \mathrm{O}: 5 / 5$, Kagi-Mosher) 0.38; ${ }^{1} \mathrm{H} \mathrm{NMR}\left(\mathrm{C}_{6} \mathrm{D}_{6}, 300 \mathrm{MHz}\right) \delta 7.30(\mathrm{~d}, J 15.5$ $\mathrm{Hz}, 1 \mathrm{H}), 6.03(\mathrm{~d}, J 9.8 \mathrm{~Hz}, 1 \mathrm{H}), 5.97(\mathrm{~d}, J 15.5 \mathrm{~Hz}, 1 \mathrm{H}), 5.63(\mathrm{~d}, J 9.2 \mathrm{~Hz}, 1 \mathrm{H}), 4.52-4.43(\mathrm{~m}, 1 \mathrm{H}), 4.32-4.22(\mathrm{~m}$, $1 \mathrm{H}), 4.11(\mathrm{dd}, J 3.6,0.9 \mathrm{~Hz}, 1 \mathrm{H}), 3.95-3.85(\mathrm{~m}, 1 \mathrm{H}), 3.70-3.64(\mathrm{~m}, 2 \mathrm{H}), 3.39(\mathrm{~s}, 3 \mathrm{H}), 3.24-3.12(\mathrm{~m}, 1 \mathrm{H}), 2.85$ $-2.71(\mathrm{~m}, 1 \mathrm{H}), 2.50(\mathrm{dd}, J 14.7,7.7 \mathrm{~Hz}, 1 \mathrm{H}), 2.37$ (dd, J 14.7, 4.6 Hz, 1H), $2.11-1.98(\mathrm{~m}, 1 \mathrm{H}), 1.89-1.77(\mathrm{~m}$, $1 \mathrm{H}), 1.77-1.68(\mathrm{~m}, 1 \mathrm{H}), 1.68-1.59(\mathrm{~m}, 6 \mathrm{H}), 1.46(\mathrm{~m}, 2 \mathrm{H}), 1.25(\mathrm{~s}, 3 \mathrm{H}), 1.24(\mathrm{~s}, 3 \mathrm{H}), 1.18(\mathrm{dd}, \mathrm{J} 6.8,5.4 \mathrm{~Hz}, 6 \mathrm{H})$, $1.11(\mathrm{~d}, J 7.1 \mathrm{~Hz}, 3 \mathrm{H}), 1.04(\mathrm{~s}, 9 \mathrm{H}), 1.02(\mathrm{~s}, 9 \mathrm{H}), 1.01(\mathrm{~s}, 9 \mathrm{H}), 0.98(\mathrm{~s}, 9 \mathrm{H}), 0.97-0.94(\mathrm{~m}, 3 \mathrm{H}), 0.94-0.90(\mathrm{~m}$, $6 \mathrm{H}), 0.89(\mathrm{~d}, J 5.6 \mathrm{~Hz}, 3 \mathrm{H}), 0.25(\mathrm{~s}, 3 \mathrm{H}), 0.19(\mathrm{~s}, 3 \mathrm{H}), 0.16(\mathrm{~s}, 3 \mathrm{H}), 0.16(\mathrm{~s}, 3 \mathrm{H}), 0.15(\mathrm{~s}, 3 \mathrm{H}), 0.14(\mathrm{~s}, 3 \mathrm{H}), 0.12(\mathrm{~s}$, $3 \mathrm{H}), 0.10(\mathrm{~s}, 3 \mathrm{H}) ;{ }^{13} \mathrm{C}$ NMR (major isomer) $\left(100 \mathrm{MHz}, \mathrm{C}_{6} \mathrm{D}_{6}\right) \delta 211.9,171.7,165.5,149.2,139.1,128.3,123.1$, 83.7, 79.5, 78.9, 76.1, 70.4, 54.6, 51.3, 51.0, 45.1, 42.7, 40.0, 36.2, 35.2, 31.6, 31.4, 30.2, 28.8, 26.7, 26.4, 26.3, $26.1,23.9,23.0,20.0,19.9,18.9,18.8,18.3,18.0,16.7,16.6,16.6,16.3,11.1,-2.6,-2.8,-3.4(2 \mathrm{C}),-3.7,-4.2$, $-4.6(2 \mathrm{C}) . \mathrm{MS}\left(\mathrm{ESI}: \mathrm{NH}_{3}\right) \mathrm{m} / \mathrm{z} 1093[\mathrm{M}+\mathrm{Na}]^{+}, 1088\left[\mathrm{M}+\mathrm{NH}_{4}\right]^{+}$.

Methyl $(3 R, 6 S, 10 R, 11 R, 12 R, 13 R)(E)-3,11,13-t r i s[(t e r t-b u t y l d i m e t h y l s i l y l) o x y]-12-\{(6 S, 7 S)(E)-7-[(t e r t-b u t y l-$ dimethylsilyl)oxy]-4,4,6,8-tetramethyl-5-oxonon-2-enamido\}-7-hydroxy-6,8,10,14-tetramethylpentadec-8enoate (36). Compound 36 was obtained in 34\% yield as a colorless oil from 34 (112 mg, 0.125 mmol, 1.2 equiv) and (R,S)-33 (32 mg, $0.104 \mathrm{mmol}, 1$ equiv) following the same procedure than for compound $35 .{ }^{1} \mathrm{H}$ NMR (major isomer) (400 MHz, C6 $\left.\mathrm{D}_{6}\right) \delta 7.36(\mathrm{~d}, J 15.5 \mathrm{~Hz}, 1 \mathrm{H}), 6.09(\mathrm{~d}, J 9.7 \mathrm{~Hz}, 1 \mathrm{H}), 5.97(\mathrm{~d}, J 15.5 \mathrm{~Hz}, 1 \mathrm{H})$, $5.42(\mathrm{~d}, J 10.2 \mathrm{~Hz}, 1 \mathrm{H}), 4.23(\mathrm{~d}, J 2.1 \mathrm{~Hz}, 2 \mathrm{H}), 4.15(\mathrm{t}, J 9.6 \mathrm{~Hz}, 1 \mathrm{H}), 3.92(\mathrm{dd}, J$ J.2, $1.7 \mathrm{~Hz}, 1 \mathrm{H}), 3.62(\mathrm{dd}, J 9.6,1.5$ $\mathrm{Hz}, 1 \mathrm{H}), 3.55(\mathrm{~d}, J 9.3 \mathrm{~Hz}, 1 \mathrm{H}), 3.36(\mathrm{~s}, 3 \mathrm{H}), 3.21(\mathrm{dd}, J 9.2,6.8 \mathrm{~Hz}, 1 \mathrm{H}), 2.78-2.73(\mathrm{~m}, 1 \mathrm{H}), 2.46(\mathrm{dd}, J 14.8,7.7$ $\mathrm{Hz}, 1 \mathrm{H}), 2.26(\mathrm{dd}, J 14.8,4.7 \mathrm{~Hz}, 1 \mathrm{H}), 2.03-2.00(\mathrm{~m}, 2 \mathrm{H}), 1.83(\mathrm{~d}, J 1.2 \mathrm{~Hz}, 3 \mathrm{H}), 1.77-1.72(\mathrm{~m}, 1 \mathrm{H}), 1.36(\mathrm{~s}, 3 \mathrm{H})$, $1.27(\mathrm{~s}, 3 \mathrm{H}), 1.18-1.14(\mathrm{~m}, 11 \mathrm{H}), 1.10(\mathrm{~d}, J 7.1 \mathrm{~Hz}, 3 \mathrm{H}), 1.02(\mathrm{~s}, 18 \mathrm{H}), 0.995(\mathrm{~s}, 9 \mathrm{H}), 0.991(\mathrm{~s}, 9 \mathrm{H}), 1.02-0.90$ $(\mathrm{m}, 12 \mathrm{H}), 0.18(\mathrm{~s}, 3 \mathrm{H}), 0.17(\mathrm{~s}, 3 \mathrm{H}), 0.15(\mathrm{~s}, 6 \mathrm{H}), 0.15(\mathrm{~s}, 3 \mathrm{H}), 0.13(\mathrm{~s}, 3 \mathrm{H}), 0.12(\mathrm{~s}, 3 \mathrm{H}), 0.11(\mathrm{~s}, 3 \mathrm{H}) ;{ }^{13} \mathrm{C} \mathrm{NMR}$ (major isomer) $\left(100 \mathrm{MHz}, \mathrm{C}_{6} \mathrm{D}_{6}\right) \delta 211.9,171.7,165.5,149.2,139.1,128.7,123.1,83.9,79.5,78.9,76.1,70.3$, 54.6, 51.3, 51.0, 45.1, 42.7, 40.0, 36.5, 35.4, 32.4, 31.6, 31.4, 30.2, 30.0, 29.8, 28.7, 26.7, 26.4, 26.3, 26.1, 23.9, 23.1, 23.0, 19.9, 18.9, 18.8, 18.0, 16.3, 14.4, 11.1, -2.6, -2.8, -2.8, -3.5, -3.7, -4.2, -4.5, -4.6; MS (ESI, NH3) : $\mathrm{m} / \mathrm{z} 1071[\mathrm{M}+\mathrm{H}]^{+}, 1093[\mathrm{M}+\mathrm{Na}]^{+}, 1088\left[\mathrm{M}+\mathrm{NH}_{4}\right]^{+}$.

\section{Acknowledgements}

We thank the Agence Nationale de la Recherche for financial support (ANR STIMAS, 2011-2014, J.C. and P.G.E.).

\section{References}

1. Bewley, C. A.; Faulkner, D. J. Angew. Chem Int. Ed. 1998, 37, 2162.

https://doi.org/10.1002/(SICl)1521-3773(19980904)37:16<2162::AID-ANIE2162>3.0.CO;2-2

2. Winder, P. L.; Pomponi, S. A.; Wright, A. E. Mar. Drugs 2011, 9, 2643. 
https://doi.org/10.3390/md9122643

3. Molinski, T. F.; Dalisay, D. S.; Lievens, S. L.; Saludes, J. P. Nature Rev. Drug Discovery 2009, 8, 69. https://doi.org/10.1038/nrd2487

4. Blunt, J. W.; Copp, B. R.; Munro, M. H. G.; Northcote, T. Nat. Prod. Rep. 2010, 27, 165. https://doi.org/10.1039/b906091j

5. Vinothkumar, S.; Parameswaran, P. S. Biotechnol. Adv. 2013, 31, 1826. https://doi.org/10.1016/j.biotechadv.2013.02.006

6. Matsunaga, S.; Fusetani, N.; Kato, Y.; Hirota, H. J. Am. Chem. Soc. 1991, 113, 9690. https://doi.org/10.1021/ja00025a054

7. Youssef, D.T.; Mooberry, S.L. J. Nat. Prod. 2006, 69, 154. https://doi.org/10.1021/np050404a

8. Plaza, A.; Baker, H. L.; Bewley, C. A. J. Nat. Prod. 2008, 71, 473-477. Correction: Plaza, A.; Baker, H. L.; Bewley, C. A. J. Nat. Prod. 2009, 72, 324. https://doi.org/10.1021/np800736v

9. Marine sponge picture is by courtesy and copyright of Patrick Colin and Lori Bell, Coral Reef Research Foundation, Republic of Palau.

10. Prévost, S.; Gauthier, S.; Cano de Andrade, M. C.; Mordant, C.; Touati, A. R.; Lesot, P.; Savignac, P.; Ayad, T.; Phansavath, P.; Ratovelomanana-Vidal, V.; Genet, J.-P. Tetrahedron: Asymmetry 2010, 21, 1436. https://doi.org/10.1016/j.tetasy.2010.05.017

11. Prévost, S.; Ayad, T.; Phansavath, P.; Ratovelomanana-Vidal, V. Adv. Synth. Catal. 2011, 353, 3213. https://doi.org/10.1002/adsc.201100579

12. Perez, M.; Echeverria, P.-G.; Martinez-Arripe, E.; Ez Zoubir, M.; Touati, R.; Zhang, Z.; Genet, J.; Phansavath, P.; Ayad, T.; Ratovelomanana-Vidal, V. Eur. J. Org. Chem. 2015, 5949. https://doi.org/10.1002/ejoc.201500661

13. Perez, M.; Ayad, T.; Maillos, P.; Poughon, V.; Fahy, J.; Ratovelomanana-Vidal, V. ACS Med. Chem. Lett. 2016, 7, 403. https://doi.org/10.1021/acsmedchemlett.5b00441

14. Cossy, J.; Willis, C.; Bellosta, V.; Saint-Jalmes, L. Synthesis 2002, 7, 951. https://doi.org/10.1055/s-2002-28505

15. Cossy, J.; Dumas, C.; Gomez Pardo, D. Synlett 1997, 8, 905. https://doi.org/10.1055/s-1997-5789

16. Bensoussan, C.; Rival, N.; Hanquet, G.; Colobert, F.; Reymond, S.; Cossy, J. Tetrahedron 2013, 69, 7759. https://doi.org/10.1016/j.tet.2013.05.067

17. Guérinot, A.; Lepesqueux, G.; Sablé, S.; Reymond, S.; Cossy, J. J. Org. Chem. 2010, 75, 5151. https://doi.org/10.1021/jo100871m

18. Echeverria, P.-G.; Prévost, S.; Cornil, J.; Férard, C.; Reymond, S.; Guérinot, A.; Cossy, J.; RatovelomananaVidal, V.; Phansavath, P. Org. Lett. 2014, 16, 2390. https://doi.org/10.1021/ol500720j

19. Cornil, J.; Echeverria, P.-G.; Reymond, S.; Phansavath, P.; Ratovelomanana-Vidal, V.; Guérinot, A.; Cossy, J. Org. Lett. 2016, 18, 4534. https://doi.org/10.1021/acs.orglett.6b02162

20. Cornil, J.; Echeverria, P.-G.; Phansavath, P.; Ratovelomanana-Vidal, V.; Guérinot, A.; Cossy, J. Org. Lett. 2015, 17, 948. 
21. Makino, K.; Goto, T.; Hiroki, Y.; Hamada, Y. Angew. Chem. Int. Ed. 2004, 43, 882.

https://doi.org/10.1002/anie.200353072

22. Mordant, C.; Dünkelmann, P.; Ratovelomanana-Vidal, V.; Genêt, J.-P. Chem. Commun. 2004, 1296.

23. Mordant, C.; Dünkelmann, P.; Ratovelomanana-Vidal, V.; Genêt, J.-P. Eur. J. Org. Chem. 2004, 3017.

24. Noyori, R.; Tokunaga, M.; Kitamura, M. Bull. Chem. Soc. Jpn. 1995, 68, 36.

https://doi.org/10.1246/bcsj.68.36

25. Pellissier, H. Tetrahedron 2003, 59, 8291.

https://doi.org/10.1016/S0040-4020(03)01022-6

26. Echeverria, P.-G.; Ayad, T.; Phansavath, P.; Ratovelomanana-Vidal, V. Synthesis 2016, 48, 2523. https://doi.org/10.1055/s-0035-1561648

27. Noyori, R. Angew. Chem. Int. Ed. 2002, 41, 2008. https://doi.org/10.1002/1521-3773(20020617)41:12<2008::AID-ANIE2008>3.0.CO;2-4

28. Yoshimura, M.; Tanaka, S.; Kitamura, M. Tetrahedron Lett. 2014, 55, 3635. https://doi.org/10.1016/i.tetlet.2014.04.129

29. Xie, J.-H.; Bao, D.-H.; Zhou, Q.-L. Synthesis 2015, 47, 460.

https://doi.org/10.1055/s-0034-1378939

30. Jeulin, S.; Champion, N.; Dellis, P.; Ratovelomanana-Vidal, V.; Genêt, J.-P. Synthesis 2005, 3666.

31. Seco, J. M.; Quiñoá, E.; Riguera, R. Chem. Rev. 2012, 112, 4603.

https://doi.org/10.1021/cr2003344

32. Mitsunobu, O.; Yamada, M. Bull. Chem. Soc. Jpn. 1967, 40, 2380.

https://doi.org/10.1246/bcsj.40.2380

33. Kumara Swamy, K. C.; Bhuvan Kumar, N. N.; Balaraman, E.; Pavan Kumar, K. V. P. Chem. Rev. 2009, 109, 2551.

https://doi.org/10.1021/cr800278z

34. Blakemore, P. R. J. Chem. Soc., Perkin Trans. 1 2002, 2563.

https://doi.org/10.1039/b208078h

35. See the experimental section.

36. Ikariya, T.; Blacker, J. Acc. Chem. Res., 2007, 40, 1300. https://doi.org/10.1021/ar700134q

37. Wang, D.; Astruc, D. Chem. Rev., 2015, 115, 6621.

https://doi.org/10.1021/acs.chemrev.5b00203

38. Foubelo, F.; Najera, C.; Yus, M. Tetrahedron: Asymmetry 2015, $26,769$.

https://doi.org/10.1016/j.tetasy.2015.06.016

39. Zheng, L.-S.; Férard, C.; Phansavath, P.; Ratovelomanana-Vidal, V. Chem. Commun. 2018, 54, 283. https://doi.org/10.1039/C7CC08231B

40. Zheng, L.-S.; Phansavath, P.; Ratovelomanana-Vidal, V. Org. Lett. 2018, 20, 5107. https://doi.org/10.1021/acs.orglett.8b01943

41. Matsumura, K.; Hashiguchi, S.; Ikariya, T.; Noyori, R. J. Am. Chem. Soc. 1997, 119, 8738. https://doi.org/10.1021/ja971570a

42. Hayes, A. M.; Morris, D. J.; Clarkson, G. J.; Wills, M. J. Am. Chem. Soc. 2005, 127, 7318. https://doi.org/10.1021/ja051486s

43. Echeverria, P.-G.; Férard, C.; Phansavath, P.; Ratovelomanana-Vidal, V. Catal. Commun. 2015, $62,95$. https://doi.org/10.1016/j.catcom.2015.01.012 
44. Zheng, L.-S.; Llopis, Q.; Echeverria, P.-G.; Férard, C.; Guillamot, G.; Phansavath, P.; Ratovelomanana-Vidal, V. J. Org. Chem. 2017, 82, 5607.

https://doi.org/10.1021/acs.joc.7b00436

45. Garegg, P. J.; Samuelsson, B. J. Chem. Soc., Chem. Commun. 1979, 978.

https://doi.org/10.1039/c39790000978

46. Weiler, L. J. Am. Chem. Soc. 1970, 92, 6702.

https://doi.org/10.1021/ja00725a088

47. Takai, K.; Tagashira, M.; Kuroda, T.; Oshima, K.; Utimoto, K.; Nozaki, H. J. Am. Chem. Soc. 1986, $108,6048$. https://doi.org/10.1021/ja00279a068

48. Harnying, W.; Kaiser, A.; Klein, A.; Berkessel, A. Chem. Eur. J. 2011, 17, 4765.

https://doi.org/10.1002/chem.201003366 